\title{
Notes on Non-Generic Isomonodromy Deformations
}

\author{
Davide GUZZETTI
}

SISSA, Via Bonomea 265 - 34136 Trieste, Italy

E-mail: guzzetti@sissa.it

Received April 17, 2018, in final form August 14, 2018; Published online August 21, 2018

https://doi.org/10.3842/SIGMA.2018.087

\begin{abstract}
Some of the main results of [Cotti G., Dubrovin B., Guzzetti D., Duke Math. J., to appear, arXiv:1706.04808], concerning non-generic isomonodromy deformations of a certain linear differential system with irregular singularity and coalescing eigenvalues, are reviewed from the point of view of Pfaffian systems, making a distinction between weak and strong isomonodromic deformations. Such distinction has a counterpart in the case of Fuchsian systems, which is well known as Schlesinger and non-Schlesinger deformations, reviewed in Appendix A.
\end{abstract}

Key words: isomonodromy deformations; Stokes phenomenon; Pfaffian system; coalescing eigenvalues; Schlesinger deformations

2010 Mathematics Subject Classification: 34M56; 34M35; 34M40

\section{Introduction}

These notes partly touch the topics of my talk in Ann Arbor at the conference in memory of Andrei Kapaev, August 2017. They are a reworking of some of the main results of [11], concerning non-generic isomonodromy deformations of the differential system (2.1) below. The approach here is different from [11], since I will start from the point of view of Pfaffian systems. This allows to introduce the main theorem (Theorem 4.9 below) in a relatively simple way (provided we give for granted another result of [11] summarised in Theorem 4.7 below). The approach here is also an opportunity to review the difference between weak and strong isomonodromy deformations.

In Section 2, we define "weak" isomonodromic deformations of the system (2.1), through the Pfaffian system (2.2) below, that we characterise together with its fundamental matrix solutions. The residue matrix $A(u)$ is not assumed to be diagonalizable or non-resonant, so that a first non-generic issue is included in the discussion. In Section 3, we define and characterise "strong" isomonodromy deformations of the differential system (2.1) in terms of solutions of the Pfaffian system and in terms of essential monodromy data. We recover the total differential system used in the last part of [11], a special case of which (for $A(u)$ skew-symmetric) has been well known in the theory of Frobenius manifolds [16, 18]. In Section 4, we explain some of the main results of [11], particularly Theorem 4.9, which extend strong isomonodromy deformations to the non-generic case when the matrix $\Lambda=\operatorname{diag}\left(u_{1}, \ldots, u_{n}\right)$ in system $(2.1)$ has coalescing eigenvalues $\left(u_{i}-u_{j}\right) \rightarrow 0$ for some $1 \leq i \neq j \leq n$. The proof of Theorem 4.9 is based on Theorem 4.7, which holds for a differential system more general than (2.1), not necessarily isomonodromic (see system (4.7)). Paper [11] is unavoidably long because of a careful set up for the background and the proof of Theorem 4.7. Given it for granted, in these notes we can introduce and prove Theorem 4.9 in a relatively short manner, starting from the discussion of the Pfaffian system (2.2).

This paper is a contribution to the Special Issue on Painlevé Equations and Applications in Memory of Andrei Kapaev. The full collection is available at https://www.emis.de/journals/SIGMA/Kapaev.html 
At the end of these notes, I would like to show how the notion of weak and strong isomonodromy deformations also arises naturally in the framework of Fuchsian systems; in this case, "weak" and "strong" deformations respectively coincide with non-Schlesinger and Schlesinger deformations. We will review this issue in Appendix A.

Several detailed comments on the existing literature concerning non-generic isomonodromy deformations have been included in the introduction of [11], so we do not repeat them here. However, a reference is missing from the cited introduction (in the arXiv version), which I will include here in Remark 4.11 of Section 4 below.

\section{A Pfaffian system defining weak isomonodromic deformations}

In this section, particularly in Proposition 2.6, we characterise a Pfaffian system responsible for the (weak) isomonodromic deformations of the differential system

$$
\partial_{z} Y=\left(\Lambda+\frac{A(u)}{z}\right) Y
$$

The above appears naturally as the (inverse) Laplace transform of a Fuchsian system of Okubo type, with poles at $u_{1}, \ldots, u_{n}[5,19,23,31]$. A particular case of $(2.1)$ is at the core of the isomonodromic deformation approach to Frobenius manifolds [16, 18].

Consider an $n \times n$ matrix Pfaffian system

$$
\mathrm{d} Y=\omega Y, \quad \omega=\omega_{0}(z, u) \mathrm{d} z+\sum_{j=1}^{n} \omega_{j}(z, u) \mathrm{d} u_{j} .
$$

The complex $n+1$ variables will be denoted by $(z, u)$, with $u:=\left(u_{1}, \ldots, u_{n}\right)$. We assume that

$$
\omega_{0}=\Lambda+\frac{A(u)}{z}
$$

with $\Lambda=\operatorname{diag}\left(u_{1}, \ldots, u_{n}\right)$ and $A(u)$ an $n \times n$ matrix, so that $(2.2)$ can be viewed as a deformation of the differential system (2.1). We suppose that $\omega_{1}, \ldots, \omega_{n}$ are holomorphic of $(z, u) \in \mathbb{C} \times \mathbb{D}\left(u^{0}\right)$, where $\mathbb{D}\left(u^{0}\right)$ is a polydisc centred at $u^{0}=\left(u_{1}^{0}, \ldots, u_{n}^{0}\right)$, contained in

$$
\mathbb{C}^{n} \backslash \bigcup_{i \neq j}\left\{u_{i}-u_{j}=0\right\}
$$

We also assume that $A(u)$ is holomorphic on $\mathbb{D}\left(u^{0}\right)$ and that $z=\infty$ is at most a pole of the $\omega_{j}(z, u)$, so that $(2.2)$ is meromorphic on $\mathbb{P}^{1} \times \mathbb{D}\left(u^{0}\right)$. The complete Frobenius integrability of the system (2.2) is expressed by

$$
\mathrm{d} \omega=\omega \wedge \omega
$$

namely, letting $\left(x_{0}, x_{1}, \ldots, x_{n}\right):=\left(z, u_{1}, \ldots, u_{n}\right)$,

$$
\frac{\partial \omega_{\beta}}{\partial x_{\alpha}}+\omega_{\beta} \omega_{\alpha}=\frac{\partial \omega_{\alpha}}{\partial x_{\beta}}+\omega_{\alpha} \omega_{\beta}, \quad \alpha \neq \beta=0,1, \ldots, n .
$$

If the integrability condition holds [7, 24, 26, 34], then (2.2) admits of a fundamental matrix solution $Y(z, u)$, which is holomorphic on $\mathcal{R} \times \mathbb{D}\left(u^{0}\right)$, where

$\mathcal{R}:=$ the universal covering of $\mathbb{P}^{1} \backslash\{0, \infty\}$. 
Its monodromy matrix $M$ associated with a simple loop $\gamma$ around $z=0$, defined by

$$
Y(\gamma z, u)=Y(z, u) M
$$

is independent of $u$ (and of course of $z$ ). The notation above means that $\gamma$ transforms $z \in \mathcal{R}$ to $\gamma z \in \mathcal{R}$ ( $z$ and $\gamma z$ are in the same fibre over a point of $\mathbb{P}^{1} \backslash\{0, \infty\}$, which we still denote by $z$, to avoid heavy notations). To show that $M$ is constant, observe that $Y(\gamma z, u)$ is a solution (it is $Y(\cdot, u)$ seen as function on $\mathcal{R}$ evaluated at $\gamma z)$. Therefore,

$$
\begin{aligned}
\omega & =\mathrm{d} Y(\gamma z, u) Y(\gamma z, u)^{-1}=\mathrm{d} Y(z, u) Y(z, u)^{-1}+Y(z, u)\left(\mathrm{d} M M^{-1}\right) Y(z, u)^{-1} \\
& =\omega+Y(z, u)\left(\mathrm{d} M M^{-1}\right) Y(z, u)^{-1} \Longleftrightarrow \mathrm{d} M=0 .
\end{aligned}
$$

In order to avoid heavy notations, we use the letter "d" either for the differential of a function $f(z, u)$ of variables $(z, u)$ (like $\mathrm{d} Y$ above), and for the differential of a function of $u$ alone (like $\mathrm{d} M$ above).

Definition 2.1. We call (2.1), regarded as the " $z$-component" of a Pfaffian system (2.2), a weak isomonodromic family of differential systems, and $Y(z, u)$ a weak isomonodromic family of fundamental matrix solutions.

By "weak" we mean that the monodromy matrix $M$ is constant, but other monodromy data, to be introduced below, may be not.

Lemma 2.2. Let $\omega_{0}$ be as in (2.3), let $\omega_{1}, \ldots, \omega_{n}$ be holomorphic of $(z, u) \in \mathbb{C} \times \mathbb{D}\left(u^{0}\right)$, and $A(u)$ holomorphic on $\mathbb{D}\left(u^{0}\right)$. The integrability condition (2.4) implies that A satisfies

$$
\frac{\partial A}{\partial u_{j}}=\left[\omega_{j}(0, u), A\right], \quad j=1, \ldots, n,
$$

and that the above is Frobenius integrable. Moreover, the system

$$
\mathrm{d} G=\left(\sum_{j=1}^{n} \omega_{j}(0, u) \mathrm{d} u_{j}\right) G
$$

is Frobenius integrable.

Proof. Condition (2.4) for $\beta=0$ and $\alpha=j \geq 1$ yields

$$
\frac{\partial}{\partial u_{j}}\left(\Lambda+\frac{A}{z}\right)+\left(\Lambda+\frac{A}{z}\right) \omega_{j}(z, u)=\frac{\partial \omega_{j}(z, u)}{\partial z}+\omega_{j}(z, u)\left(\Lambda+\frac{A}{z}\right) .
$$

Since $\omega_{j}(z, u)=\omega(0, u)+$ Taylor series in $z$, identification of the coefficients of $z^{-1}$ yields (2.7). The condition (2.4) for $\beta=i \geq 1$ and $\alpha=j \geq 1$ is regular at $z=0$. In particular, for $z=0$ we have

$$
\frac{\partial \omega_{i}(0, u)}{\partial u_{j}}+\omega_{i}(0, u) \omega_{j}(0, u)=\frac{\partial \omega_{j}(0, u)}{\partial u_{i}}+\omega_{j}(0, u) \omega_{i}(0, u), \quad i \neq j=1, \ldots, n,
$$

which is the Frobenius integrability condition for (2.8). It is also the integrability condition of (2.7). Indeed, let us compute (for brevity, we write $\omega_{j}(0):=\omega_{j}(0, u)$ )

$$
\begin{aligned}
\partial_{k}\left[\omega_{j}(0), A\right]-\partial_{j}\left[\omega_{k}(0), A\right]=\left(\partial_{k} \omega_{j}(0) A+\omega_{j}(0) \partial_{k} A-\partial_{k} A \omega_{j}(0)-A \partial_{k} \omega_{j}(0)\right) \\
\quad-\left(\partial_{j} \omega_{k}(0) A+\omega_{k}(0) \partial_{j} A-\partial_{j} A \omega_{k}(0)-A \partial_{j} \omega_{k}(0)\right) \\
=\left(\partial_{k} \omega_{j}(0) A+\omega_{j}(0)\left[\omega_{k}(0) A-A \omega_{k}(0)\right]-\left[\omega_{k}(0) A-A \omega_{k}(0)\right] \omega_{j}(0)-A \partial_{k} \omega_{j}(0)\right)
\end{aligned}
$$




$$
\begin{aligned}
& -\left(\partial_{j} \omega_{k}(0) A+\omega_{k}(0)\left[\omega_{j}(0) A-A \omega_{j}(0)\right]-\left[\omega_{j}(0) A-A \omega_{j}(0)\right] \omega_{k}(0)-A \partial_{j} \omega_{k}(0)\right) \\
= & \left\{\left[\partial_{k} \omega_{j}(0)+\omega_{j}(0) \omega_{k}(0)\right]-\left[\partial_{j} \omega_{k}(0)+\omega_{k}(0) \omega_{j}(0)\right]\right\} A \\
& +A\left\{\left[\omega_{k}(0) \omega_{j}(0)-\partial_{k} \omega_{j}(0)\right]-\left[\omega_{j}(0) \omega_{k}(0)-\partial_{j} \omega_{k}(0)\right]\right\} .
\end{aligned}
$$

The last expression is zero for any possible $A$ if and only if (2.10) holds.

The following is a standard result concerning the residue matrix at Fuchsian singularity of a Pfaffian system (see for example [24]).

Proposition 2.3. Let $\omega_{0}$ be as in $(2.3)$, let $\omega_{1}, \ldots, \omega_{n}$ be holomorphic of $(z, u) \in \mathbb{C} \times \mathbb{D}\left(u^{0}\right)$, and $A(u)$ holomorphic on $\mathbb{D}\left(u^{0}\right)$. Then, $A(u)$ is holomorphically similar to a constant (i.e., independent of $u$ ) Jordan form $J$, namely there exists a holomorphic fundamental matrix $G(u)$ of $(2.8)$ on $\mathbb{D}\left(u^{0}\right)$, such that

$$
G(u)^{-1} A(u) G(u)=J .
$$

In particular, this means that the weak isomonodromy deformation (2.1) is isospectral.

Proof. Take a fundamental matrix $\tilde{G}(u)$ of $(2.8)$, which is holomorphic on $\mathbb{D}\left(u^{0}\right)$. Then, using (2.7) and (2.8), we have

$$
\begin{aligned}
\partial_{j}\left(\tilde{G}^{-1} A \tilde{G}\right) & =\tilde{G}^{-1} A \partial_{j} \tilde{G}+\tilde{G}^{-1} \partial_{j} A \tilde{G}-\tilde{G}^{-1} \partial_{j} \tilde{G} \tilde{G}^{-1} A \tilde{G} \\
& =\tilde{G}^{-1} A \omega_{j}(0, u) \tilde{G}+\tilde{G}^{-1} \partial_{j} A \tilde{G}-\tilde{G}^{-1} \omega_{j}(0, u) A \tilde{G} \\
& =\tilde{G}^{-1}\left(\left[A, \omega_{j}(0, u)\right]+\partial_{j} A\right) \tilde{G}=0 .
\end{aligned}
$$

This implies that $\mathcal{A}:=\tilde{G}^{-1} A \tilde{G}$ is constant over $\mathbb{D}\left(u^{0}\right)$, and it has a constant Jordan form $J=\mathcal{G}^{-1} \mathcal{A G}$ for some invertible matrix $\mathcal{G}$. Thus, the desired holomorphic matrix is $G(u)=$ $\tilde{G}(u) \mathcal{G}$.

At any fixed $u$, the differential system (2.1) admits fundamental matrix solutions in Levelt form at $z=0$,

$$
Y^{(0)}(z, u)=\widehat{Y}^{(0)}(z, u) z^{D} z^{L}
$$

where

$$
\widehat{Y}^{(0)}(z, u)=G(u)\left(I+\sum_{j=1}^{\infty} \Psi_{j}(u) z^{j}\right)
$$

is a convergent matrix valued Taylor series ( $I$ stands for the identity matrix). The matrix $G(u)$ puts $A(u)$ in Jordan form $J=G(u)^{-1} A(u) G(u)$ and satisfies (2.8) as in Proposition 2.3. The following characterisation holds $[1,21]$.

- $L$ is block-diagonal $L=L_{1} \oplus \cdots \oplus L_{\ell}$, with upper triangular matrices $L_{q}$; each $L_{q}$ has only one eigenvalue $\sigma_{q}$, satisfying $0 \leq \operatorname{Re} \sigma_{q}<1$, and $\sigma_{p} \neq \sigma_{q}$ for $1 \leq p \neq q \leq \ell$.

- $D$ is a diagonal matrix of integers, which can be split into blocks $D_{1} \oplus \cdots \oplus D_{\ell}$ as $L$. The integers $d_{q, r}$ in each $D_{q}=\operatorname{diag}\left(d_{q, 1}, d_{q, 2}, \ldots\right)$ form a non-increasing (finite) sequence $d_{q, 1} \geq d_{q, 2} \geq \cdots$.

- The eigenvalues of $A$ are $d_{q, s}+\sigma_{q}$, for $q=1, \ldots, \ell$ and $s$ runs from 1 to some integer $n_{q}$, if the dimension of $L_{q}$ is $n_{q} \times n_{q}$. Each block $L_{q}(u)$ corresponds to the eigenvalues of $A(u)$ which differ by non-zero integers. 
- The expression in square brackets below is holomorphic at $z=0$ and the following limits hold

$$
\begin{aligned}
\lim _{z \rightarrow 0}\left[\widehat{Y}^{(0)}(z, u)\left(D+z^{D} L z^{-D}\right)\left(\widehat{Y}^{(0)}(z, u)\right)^{-1}\right] \\
\quad=\lim _{z \rightarrow 0}\left[G(u)\left(D+z^{D} L z^{-D}\right) G(u)^{-1}\right]=A(u) .
\end{aligned}
$$

Equivalently, $D+\lim _{z \rightarrow 0} z^{D} L z^{-D}=J$.

A Levelt form (2.11) is not uniquely determined by the differential system, since there is a freedom in the choice of the coefficients $\Psi_{j}(u)$ and the exponents $D$ and $L$. See [11] (and also [18]) for more details.

The fundamental matrices (2.11), seen as solutions of the differential system (2.1) only, may have exponents $L$ and $D$ depending on the point $u$, and the coefficients $\Psi_{j}(u)$ are not guaranteed to be holomorphic. On the other hand, the Pfaffian system (2.2) is of Fuchsian type at $z=0$ with the only singular contribution in $\omega$ coming from $\omega_{0}(z, u)=A(u) / z+\Lambda$. For this reason, system (2.2) also admits solutions of the form (2.11), behaving nicely with respect to $u \in \mathbb{D}\left(u^{0}\right)$, according to the following

Proposition 2.4. Let $A(u)$ be holomorphic on $\mathbb{D}\left(u^{0}\right)$ and $\omega_{1}(z, u), \ldots, \omega_{n}(z, u)$ be holomorphic on $\mathbb{C} \times \mathbb{D}\left(u^{0}\right)$. Then:

- the Pfaffian system (2.2) admits fundamental matrix solutions in Levelt form (2.11)-(2.12);

- the exponents $D$ and $L$ are constant;

- the convergent series (2.12) defines a holomorphic matrix valued function on $\mathbb{C} \times \mathbb{D}\left(u^{0}\right)$; equivalently, $Y^{(0)}(z, u)$ is holomorphic on $\mathcal{R} \times \mathcal{U}_{\epsilon}\left(u^{C}\right)$.

Notice that Proposition 2.4 also holds in case $\mathbb{D}\left(u^{0}\right)$ intersects $\bigcup_{i \neq j}^{n}\left\{u_{i}-u_{j}=0\right\}$, provide that $A(u)$ and $\omega_{1}, \ldots, \omega_{n}$ are holomorphic of $(z, u) \in \mathbb{C} \times \mathbb{D}\left(u^{0}\right)$ (see Corollary 4.2).

Proof. The Pfaffian system (2.2) is of Fuchsian type at $z=0$. Thus, we can apply the result of $[7,34]$. In particular, by theorem 5 of [34], there exists a gauge transformation

$$
Y=V(z, u) \tilde{Y}
$$

holomorphic in $\mathbb{C} \times \mathbb{D}\left(u^{0}\right)$ and represented by a convergent matrix valued series

$$
V(z, u)=I+\sum_{k: k_{1}+\cdots+k_{n}>0} V_{k} z^{k_{0}}\left(u-u_{1}^{0}\right)^{k_{1}} \cdots\left(u-u_{n}^{0}\right)^{k_{n}}
$$

(here $k=\left(k_{0}, k_{1}, \ldots, k_{n}\right)$ and $V_{k}$ are matrices), such that

$$
\mathrm{d} \tilde{Y}=\left[\omega_{0}\left(z, u^{0}\right) \mathrm{d} z\right] \tilde{Y} \equiv\left[\left(\frac{A\left(u^{0}\right)}{z}+\Lambda\left(u^{0}\right)\right) \mathrm{d} z\right] \tilde{Y} .
$$

The above admits fundamental solutions in Levelt form

$$
\widetilde{Y}^{(0)}(z)=W(z) z^{D} z^{L}, \quad W(z)=W_{0}+\sum_{\ell=1}^{\infty} W_{\ell} z^{\ell}, \quad \operatorname{det}\left(W_{0}\right) \neq 0,
$$

which are independent of $u$ ( $u^{0}$ being fixed). Here, $W(z)$ converges and defines a holomorphic function on $\mathbb{C}$. This means that

$$
Y^{(0)}(z, u):=V(z, u) W(z) \tilde{Y}^{(0)}(z)
$$


is a fundamental matrix solution of (2.2) in Levelt form, with constant exponents $L$ and $D$. Moreover, $V(z, u) W(z)$ is holomorphic on $\mathbb{C} \times \mathbb{D}\left(u^{0}\right)$ and its series representation can be rearranged as follows

$$
G(u)\left(I+\sum_{j=1}^{\infty} \Psi_{j}(u) z^{j}\right), \quad G(u)=V(0, u) W_{0} .
$$

Notice that $G(u)$ puts $A(u)$ in Jordan form. The proof above can be also deduced by directly applying Theorems 4 and 7 of [34].

Chosen a holomorphic isomonodromic family $Y(z, u)$ of $(2.2)$, with holomorphic $\omega_{1}, \ldots, \omega_{n}$ at $z=0$, then there exists a connection matrix $H$ such that

$$
Y(z, u)=Y^{(0)}(z, u) H \text {. }
$$

By Proposition 2.4, also $Y^{(0)}$ is an isomonodromic family of fundamental solutions, so that $H$ cannot depend on $u$. Indeed

$$
\begin{aligned}
\omega=\mathrm{d} Y Y^{-1} & =\mathrm{d} Y^{(0)}\left(Y^{(0)}\right)^{-1}+Y^{(0)} \mathrm{d} H H^{-1}\left(Y^{(0)}\right)^{-1} \\
& =\omega+Y^{(0)} \mathrm{d} H H^{-1}\left(Y^{(0)}\right)^{-1} \Longleftrightarrow \mathrm{d} H=0 .
\end{aligned}
$$

Without loss of generality, we can always choose

$$
Y(z, u)=Y^{(0)}(z, u)
$$

Remark 2.5. For the sake of computations, we can write $L=\Sigma+N$, where $\Sigma=\Sigma_{1} \oplus \cdots \oplus \Sigma_{\ell}$ is diagonal, with $\Sigma_{q}=\sigma_{q} I_{\operatorname{dim}\left(\Sigma_{q}\right)}$ (here $I_{\operatorname{dim}\left(\Sigma_{q}\right)}$ stands for the identity matrix of dimension $\operatorname{dim}\left(\Sigma_{q}\right)$ ), while $N=N_{1} \oplus \cdots \oplus N_{\ell}$ is nilpotent. Since $\Sigma_{q}$ and $N_{q}$ have the same dimension $(q=1, \ldots, \ell)$, we have

$$
[\Sigma, N]=0
$$

and thus

$$
z^{D} z^{L}=z^{D} z^{\Sigma} z^{N}=z^{D+\Sigma} z^{N}=z^{D+\Sigma} \sum_{\ell=0}^{\bar{k}} \frac{N^{\ell}}{\ell !}(\log z)^{\ell},
$$

for some finite $\bar{k}$.

The differential system (2.1) also admits a family of fundamental matrices $Y_{r}(z, u), r \in \mathbb{Z}$, uniquely determined by their asymptotic behaviour in suitable sectors of central angular opening greater that $\pi$. We fix a half-line of direction $\arg z=\tau$ in $\mathcal{R}$ which does not coincide with any of the Stokes rays associated with $\Lambda\left(u^{0}\right)$, namely with the half lines in $\mathcal{R}$ specified by

$$
\operatorname{Re}\left(z\left(u_{i}^{0}-u_{j}^{0}\right)\right)=0, \quad \operatorname{Im}\left(z\left(u_{i}^{0}-u_{j}^{0}\right)\right)<0 .
$$

Notice that in order to obtain half-lines, we need to specify the sign the imaginary part. We say that $\tau$ is an admissible direction at $u^{0}$. Notice that we are working in the universal covering $\mathcal{R}$, so that any $\tau+h \pi, h \in \mathbb{Z}$ is also an admissible direction. We define the Stokes rays associated with $\Lambda(u)$ to be the infinitely many half-lines in $\mathcal{R}$ defined by

$$
\operatorname{Re}\left(z\left(u_{i}-u_{j}\right)\right)=0, \quad \operatorname{Im}\left(z\left(u_{i}-u_{j}\right)\right)<0 .
$$


These rays rotate as $u$ varies in $\mathbb{D}\left(u^{0}\right)$, and may cross an admissible direction. Let $\mathbb{D}^{\prime} \subset \mathbb{D}\left(u^{0}\right)$ be a disk sufficiently small so that no Stokes rays cross a direction $\tau+h \pi$ as $u$ varies in the closure of $\mathbb{D}^{\prime}$. Then, for $u \in \overline{\mathbb{D}^{\prime}}$, we construct a sector $\mathcal{S}_{1}(u)$, by considering the "half plane" $\tau-\pi<\arg z<\tau$ and extending it to the nearest Stokes rays associated with $\Lambda(u)$ lying outside the half plane. Analogously, for any $r \in \mathbb{Z}$ a sector $\mathcal{S}_{r}(u)$ is obtained extending the "half plane" $\tau+(r-2) \pi<\arg z<\tau+(r-1) \pi$ to the nearest Stokes rays outside of it. The sectors $\mathcal{S}_{r}(u)$ have central angular opening greater than $\pi$, and the same holds for

$$
\mathcal{S}_{r}\left(\overline{\mathbb{D}^{\prime}}\right):=\bigcap_{u \in \overline{\mathbb{D}^{\prime}}} \mathcal{S}_{r}(u)
$$

Since $u_{i} \neq u_{j}$ for $i \neq j$, it is well known that the system (2.1) has a formal fundamental matrix solution

$$
Y_{F}(z, u)=F(z, u) z^{B(u)} e^{z \Lambda},
$$

where

$$
B(u):=\operatorname{diag}\left(A_{11}(u), \ldots, A_{n n}(u)\right)
$$

is a diagonal matrix, and $F(z, u)$ is the formal matrix-valued expansion

$$
F(z, u)=I+\sum_{k=1}^{\infty} F_{k}(u) z^{-k}
$$

with holomorphic on $\mathbb{D}\left(u^{0}\right)$ coefficients $F_{k}(u)$ uniquely determined by (2.1). Moreover, for each fixed $u$, and for any sector $S$ containing in its interior a set of basic Stokes rays ${ }^{1}$ and no other Stokes rays, it is well known [2] that there exists a unique fundamental matrix solution

$$
Y_{(S)}(z, u)=\widehat{Y}_{(S)}(z, u) z^{B(u)} e^{z \Lambda}
$$

such that

$$
\widehat{Y}_{(S)}(z, u) \sim F(z, u), \quad z \rightarrow \infty \text { in } S .
$$

Now, if $u$ is restricted to $\mathbb{D}^{\prime}$, then we can take a family of such solutions, labelled by $r \in \mathbb{Z}$, with $S=\mathcal{S}_{r}(u)$,

$$
\begin{aligned}
& Y_{r}(z, u)=\widehat{Y}_{r}(z, u) z^{B(u)} e^{z \Lambda}, \\
& \widehat{Y}_{r}(z, u) \sim F(z, u), \quad z \rightarrow \infty \text { in } \mathcal{S}_{r}(u) .
\end{aligned}
$$

It is a fundamental result of Sibuya's $[25,32,33]$ that each $Y_{r}(z, u)$ depends holomorphically on $u \in \mathbb{D}^{\prime}$, and the asymptotics (2.18) is uniform for $z \rightarrow \infty$ in $\mathcal{S}_{r}\left(\overline{\mathbb{D}^{\prime}}\right)$ with respect to $u$ varying in $\mathbb{D}^{\prime}$. We notice that it may be necessary to further restrict $\mathbb{D}^{\prime}$, because Sibuya's result requires $\mathbb{D}^{\prime}$ to

${ }^{1}$ Let $\mu$ be an integer. We say that a finite sequence of Stokes rays $\arg z=\tau_{1}, \quad \arg z=\tau_{2}, \quad \ldots, \quad \arg z=\tau_{\mu}, \quad \tau_{1}<\tau_{2}<\cdots<\tau_{\mu}, \quad\left(\tau_{\mu}-\tau_{1}\right)<\pi$,

form a set of basic Stokes rays if all the other Stokes rays can be obtained as

$$
\arg z=\tau_{\nu}+k \pi, \quad \text { for some } \nu \in\{1,2, \ldots, \mu\} \text { and } k \in \mathbb{Z} \text {. }
$$

There exist a set of basic rays (and then infinitely many). Indeed, a sector with angular opening $\pi$, whose boundary rays are not Stokes rays, contains exactly a set of basic rays. 
be sufficiently small in order for the holomorphic dependence to occur. The Stokes matrices $\mathbb{S}_{r}$ defined by

$$
Y_{r+1}(z, u)=Y_{r}(z, u) \mathbb{S}_{r}(u),
$$

are holomorphic on $\mathbb{D}^{\prime}$. We recall that the structure of the Stokes matrices is such that $\operatorname{diag}\left(\mathbb{S}_{r}\right)=I$, and

$$
\left(\mathbb{S}_{r}\right)_{i j}=0 \quad \text { for } i, j \text { such that } e^{\left(u_{i}-u_{j}\right) z} \rightarrow \infty, \quad z \in \mathcal{S}_{r}\left(\overline{\overline{\mathbb{D}}^{\prime}}\right) \cap \mathcal{S}_{r+1}\left(\overline{\overline{\mathbb{D}}^{\prime}}\right) .
$$

This is a "triangular structure". Successive matrices $\mathbb{S}_{r}$ and $\mathbb{S}_{r+1}$ have opposite "triangular" structures.

Given the holomorphic isomonodromic family $Y(z, u)$, there will exist holomorphic connection matrices $H_{r}(u), u \in \mathbb{D}^{\prime}$, such that

$$
Y(z, u)=Y_{r}(z, u) H_{r}(u), \quad u \in \mathbb{D}^{\prime} .
$$

Let $E_{j}$ be the matrix with the only non-zero entry being $\left(E_{j}\right)_{j j}=1$. Notice that for distinct eigenvalues we have $\partial_{j} \Lambda=E_{j}$. The following proposition holds.

Proposition 2.6. Consider a completely integrable linear Pfaffian system

$$
\mathrm{d} Y=\omega Y, \quad \omega=\left(\Lambda+\frac{A(u)}{z}\right) \mathrm{d} z+\sum_{j=1}^{n} \omega_{j}(z, u) \mathrm{d} u_{j},
$$

with $A(u)$ holomorphic on $\mathbb{D}\left(u^{0}\right)$ and $\omega_{1}(z, u), \ldots, \omega_{n}(z, u)$ holomorphic on $\mathbb{C} \times \mathbb{D}\left(u^{0}\right)$. Let $z=\infty$ be at most a pole of $\omega_{1}, \ldots, \omega_{n}$. Then:

$(A)$ each $\omega_{j}(z, u)$ is linear in $z$ and determined by $A(u)$ up to an arbitrary holomorphic diagonal matrix $\mathcal{D}_{j}(u)$ on $\mathbb{D}\left(u^{0}\right)$, with the following structure ${ }^{2}$

$$
\begin{aligned}
& \omega_{j}(z, u)=z E_{j}+\omega_{j}(0, u), \\
& \omega_{j}(0, u):=\left(\frac{A_{a b}(u)\left(\delta_{a j}-\delta_{b j}\right)}{u_{a}-u_{b}}\right)_{a, b=1}^{n}+\mathcal{D}_{j}(u),
\end{aligned}
$$

where $\mathcal{D}_{j}(u)$ is obtained by differentiating a matrix $\mathcal{D}$ on $\mathbb{D}\left(u^{0}\right)$, whose diagonal entries only depend on $u$ :

$$
\mathcal{D}_{j}(u)=\frac{\partial \mathcal{D}(u)}{\partial u_{j}}
$$

(B) A(u) satisfies (2.7)

$$
\frac{\partial A}{\partial u_{j}}=\left[\omega_{j}(0, u), A\right], \quad j=1, \ldots, n
$$

$$
\begin{aligned}
& { }^{2} \text { Explicitly, } \\
& \left(\frac{A_{a b}\left(\delta_{a j}-\delta_{b j}\right)}{u_{a}-u_{b}}\right)_{a, b=1}^{n}=\left(\begin{array}{ccccc}
0 & 0 & \frac{-A_{1 j}}{u_{1}-u_{j}} & 0 & 0 \\
0 & 0 & \vdots & 0 & 0 \\
\frac{A_{j 1}}{u_{j}-u_{1}} & \cdots & 0 & \cdots & \frac{A_{j n}}{u_{j}-u_{n}} \\
0 & 0 & \vdots & 0 & 0 \\
0 & 0 & \frac{-A_{n j}}{u_{n}-u_{j}} & 0 & 0
\end{array}\right)
\end{aligned}
$$


$(C)$ the above non-linear system is Frobenius integrable;

$(D)$ the diagonal of $A$, namley the matrix $B=\operatorname{diag}\left(A_{11}, \ldots, A_{n n}\right)$ in $(2.17)$, is constant;

(E) given the relations (2.19) for a fundamental matrix $Y(z, u)$ holomorphic on $\mathcal{R} \times \mathbb{D}\left(u^{0}\right)$, then each $H_{r}$ satisfies ${ }^{3}$

$$
\frac{\partial H_{r}}{\partial u_{j}}=\mathcal{D}_{j}(u) H_{r} \quad \forall r \in \mathbb{Z}
$$

$(F)$ from $(E)$, it follows that $H_{r}(u)$ is holomorphic on $\mathbb{D}\left(u^{0}\right)$, and $Y_{r}(z, u)$ extends holomorphically on $\mathcal{R} \times \mathbb{D}\left(u^{0}\right)$.

We prove the proposition in Appendix B. We notice that in the above proposition we can choose an isomonodromic family $Y(z, u)=Y^{(0)}(z, u)$, given by a Levelt form (2.11) satisfying Proposition 2.4. We also remark that by substitution of (2.17) and (2.16) into (2.1), we find

$$
\left(F_{1}\right)_{i j}=\frac{A_{i j}}{u_{j}-u_{i}}, \quad 1 \leq i \neq j \leq n, \quad\left(F_{1}\right)_{i i}=-\sum_{j \neq i} A_{i j}\left(F_{1}\right)_{j i} .
$$

Therefore

$$
\omega_{j}(z, u)=z E_{j}+\left[F_{1}(u), E_{j}\right]+\mathcal{D}_{j}(u) .
$$

Remark 2.7. In this paper, we have assumed that each $\omega_{j}(z, u)$ is holomorphic at $z=0$. More generally, one may study a Pfaffian system with Laurent expansions at $z=0$

$$
\omega_{j}(z, u)=\sum_{m=1}^{p_{j}} \frac{\omega_{j}^{(-m)}(u)}{z^{m}}+\sum_{m=0}^{\infty} \omega_{j}^{(m)}(u) z^{m} .
$$

Here, we only remark that if $A(u)$ is non-resonant for each $u$ in the domain of interest (it means that the difference of two eigenvalues of $A(u)$ cannot be a non-zero integer), then the Frobenius integrability conditions imply that $\omega_{j}(z, u)$ is holomorphic at $z=0$, namely

$$
\omega_{j}^{\left(-p_{j}\right)}(u)=\omega_{j}^{\left(-p_{j}+1\right)}(u)=\cdots=\omega_{j}^{(-1)}(u)=0 .
$$

To see this, substitute the Laurent expansion into (2.9). We find the following recurrence relations:

From the negative powers of $z$ :

$$
\begin{aligned}
& \left(A+p_{j}\right) \omega_{j}^{\left(-p_{j}\right)}-\omega_{j}^{\left(-p_{j}\right)} A=0 ; \\
& (A+m) \omega^{(-m)}-\omega^{(-m)} A=\left[\omega^{(-m-1)}, \Lambda\right], \quad \text { for } m=p_{j}-1, p_{j}-2, \ldots, 1 ; \\
& \frac{\partial A}{\partial u_{j}}=\left[\omega^{(0)}, A\right]+\left[\omega^{(-1)}, \Lambda\right] .
\end{aligned}
$$

From the power $z^{0}$ :

$$
A \omega_{j}^{(1)}-\omega_{j}^{(1)} A-\omega_{j}^{(1)}+E_{j}=\left[\omega_{j}^{(0)}, \Lambda\right] .
$$

From the positive powers $z$ :

$$
(A-m-1) \omega_{j}^{(m+1)}-\omega_{j}^{(m+1)} A=\left[\omega_{j}^{(m)}, \Lambda\right], \quad m \geq 1 .
$$

\footnotetext{
${ }^{3}$ We have $H_{r}(u)=\exp \{\operatorname{diag}(\mathcal{D}(u))\} H_{r}^{0}$, with $H_{r}^{0}$ a constant invertible matrix.
} 
If $A$ is non-resonant, the Sylvester equation (2.23) determines $\omega_{j}^{\left(-p_{j}\right)}=0$, thus the Sylvester equations (2.24) yield $\omega_{j}^{(-m)}=0, m=p_{j}-1, p_{j}-2, \ldots, 1$. Moreover, the equations (2.27) determine $\omega_{j}^{(m+1)}$ for $m \geq 1$ in terms of $\omega_{j}^{(1)}$. In particular, each $\omega_{j}^{(m+1)}=0$ if $\omega_{j}^{(1)}$ is diagonal.

Finally, we notice that, independently of the resonance properties of $A$, equation (2.26) determines the off-diagonal entries of $\omega_{j}^{(0)}$ in terms of $\omega_{j}^{(1)}$, to be

$$
\left(\omega_{j}^{(0)}\right)_{a b}=\left(u_{a}-u_{b}\right)^{-1}\left(\left[\omega_{j}^{(1)}, A\right]+\omega_{j}^{(1)}\right)_{a b} .
$$

Moreover, the diagonal part of (2.26) yields

$$
\left(\omega_{j}^{(1)}\right)_{a a}=\delta_{j a}-\sum_{b \neq a}\left(\left(\omega_{j}^{(1)}\right)_{a b} A_{b a}-A_{a b}\left(\omega_{j}^{(1)}\right)_{b a}\right) .
$$

Under the assumptions of Proposition 2.6, we find that $\omega_{j}^{(1)}=E_{j}$ and $\omega_{j}^{(m+1)}=0$ for $m \geq 1$.

\section{Strong isomonodromic deformations}

In this section, we define strong isomonodromy deformations of the differential system (2.1), which preserve a set of monodromy data, and we characterise the Pfaffian system responsible for them and its fundamental matrix solutions. The assumption here is that $\Lambda$ has distinct eigenvalues, namely we work on the domain $\mathbb{D}\left(u^{0}\right)$ previously introduced. In the next section, we will drop this assumption.

The notion of isomonodromic deformations given by Jimbo, Miwa and Ueno in [27] is stronger than the one defined in the previous section. It requires that a set of essential monodromy data, not just the monodromy matrices, are constant. In the standard theory of [27], the matrix residue $A(u)$ at a Fuchsian singularity must be non-resonant and reducible to a diagonal form with distinct eigenvalues, namely the Levelt form $(2.11)$ is assumed to be $Y^{(0)}(z, u)=$ $\widehat{Y}^{(0)}(z, u) z^{L_{0}(u)}$, where $L_{0}$ is a diagonal matrix with distinct eigenvalues (not differing by integers). Here, we do not assume this restriction, so introducing a first non-generic feature.

Definition 3.1. Let $Y(z, u)$ be a weak isomonodromic family of fundamental matrix solutions of the Pfaffian system (2.2), or equivalenlty (2.20), with coefficients (2.22). We call (2.1) a strong isomonodromic family of differential systems, and $Y(z, u)$ a strong isomonodromic family of fundamental solutions over $\mathbb{D}\left(u^{0}\right)$, if for all $r \in \mathbb{Z}$ the connection matrices $H_{r}$ in (2.19) are independent of $u$, namely

$$
\mathrm{d} H_{r}=0 .
$$

Recall that, by Proposition 2.4, a fundamental matrix $Y^{(0)}(z, u)$ in Levelt form (2.11) satisfies the Pfaffian system. We have the following characterisation.

Proposition 3.2. The deformation is strongly isomonodromic if and only if the fundamental matrices $Y_{r}(z, u)$ of the differential system (2.1) also satisfy the Pfaffian system (2.20)

$$
\mathrm{d} Y_{r}=\omega Y_{r}, \quad \forall r \in \mathbb{Z},
$$

being the coefficients

$$
\omega_{j}(z, u)=z E_{j}+\left[F_{1}, E_{j}\right] \equiv z E_{j}+\left(\frac{A_{a b}(u)\left(\delta_{a j}-\delta_{b j}\right)}{u_{a}-u_{b}}\right)_{a, b=1}^{n} .
$$


Proof. Obvious. We have

$$
\omega=\mathrm{d} Y Y^{-1}=\mathrm{d} Y_{r} Y_{r}^{-1}+Y_{r} \mathrm{~d} H_{r} H_{r}^{-1} Y_{r}^{-1} .
$$

Therefore, $\mathrm{d} Y_{r} Y_{r}^{-1}=\omega$ if and only if $\mathrm{d} H_{r}=0$. Since $\mathrm{d} H_{r}=0$, then $\mathcal{D}_{j}=0$ in Proposition 2.6, so that $\omega_{j}$ is $(3.1)$.

It is a standard result that $\mathbb{S}_{0}$ and $\mathbb{S}_{1}$, together with $B$, suffice to generate $\mathbb{S}_{r}$ for any $r \in \mathbb{Z}$, through the well known formula [2]

$$
\mathbb{S}_{2 r}=e^{-2 r \pi i B} \mathbb{S}_{0} e^{2 r \pi i B}, \quad \mathbb{S}_{2 r+1}=e^{-2 r \pi i B} \mathbb{S}_{1} e^{2 r \pi i B} .
$$

We introduce connection matrices $C_{r}$ such that

$$
Y_{r}=Y^{(0)} C_{r} .
$$

$C_{0}$ and the Stokes matrices suffice to generate all the matrices $C_{r}$. Indeed, $Y_{r}=Y_{0} \mathbb{S}_{0} \mathbb{S}_{1} \cdots \mathbb{S}_{r-1}$, so that we have

$$
C_{r}=C_{0} \mathbb{S}_{0} \mathbb{S}_{1} \cdots \mathbb{S}_{r-1}
$$

Definition 3.3. The matrices $\mathbb{S}_{r}, B, D, L, C_{r}(r \in \mathbb{Z})$ are called the essential monodromy data associated with the fundamental matrix solutions $Y_{r}(z, u)$ and $Y^{(0)}(z, u)$ of the system (2.1). In view of (3.2) and (3.3), it suffices to consider the data

$$
\mathbb{S}_{0}, \mathbb{S}_{1}, B, D, L, C_{0}
$$

or equivalently, for some fixed value of $r$,

$$
\mathbb{S}_{r}, \mathbb{S}_{r+1}, B, D, L, C_{r}
$$

The above definition is similar to the definition of monodromy data given in [27], here including the case when $A$ may be resonant and/or non-diagonalizable.

For a Pfaffian system with $\omega_{1}, \ldots, \omega_{n}$ holomorphic on $\mathbb{C} \times \mathbb{D}\left(u^{0}\right)$, by Propositions 2.4 and 2.6, we have that

$D, L, B$ are constant on $\mathbb{D}\left(u^{0}\right)$.

The following characterisation of strong isomonodromic deformations in terms of essential monodromy data holds.

Proposition 3.4. A deformation is strongly isomonodromic as in Definition 3.1 if and only if

$$
\mathrm{d} \mathbb{S}_{r}=\mathrm{d}_{r+1}=0, \quad \mathrm{~d} C_{r}=0,
$$

for one value of $r \in \mathbb{Z}$.

Before giving the proof, we make a few comments. First, we observe that if the Proposition holds for one value of $r$, for example for $\mathbb{S}_{0}, \mathbb{S}_{1}$ and $C_{0}$, then it holds for any $r$, and conversely, by formulae (3.2) and (3.3).

Proposition 3.4 says that one can give a definition of strong isomonodromic deformations alternative to Definition 3.1, namely a deformation such that ${ }^{4}$

$D$ is constant, $\quad \mathrm{d} L=\mathrm{d} C_{0}=\mathrm{dS}_{0}=\mathrm{d} \mathbb{S}_{1}=\mathrm{d} B=0$

\footnotetext{
${ }^{4}$ We don't write $d D=0$, because $D$ has integer entries.
} 
on $\mathbb{D}\left(u^{0}\right)$. This is the definition adopted in [27], in case $A(u)$ is diagonalizable with distinct eigenvalues and no resonances. Here we have extended it without assumptions on $A(u)$. Notice also that one should, as in [27], say that $d \mathbb{S}_{0}=\mathrm{d} \mathbb{S}_{1}=\mathrm{d} C_{0}$ on the polydisc $\mathbb{D}^{\prime}$ where $Y_{r}(z, u)$ have the properties prescribed by Sibuya's results. Nevertheless, by Proposition 2.6 point (F), we can extend the properties to $\mathbb{D}\left(u^{0}\right)$.

In case $A(u)$ is skew-symmetric and diagonalizable, the above characterisation of isomonodromic deformations with form (3.1) is well known in the theory of Frobenius manifolds, developed in $[16,18]$, where $A(u)$ is named $V(u)$ and $\Lambda$ is called $U$.

Proof of Proposition 3.4. For a weak isomonodromic deformation $D, L$ and $B$ are constant (Propositions 2.4 and 2.6), so we have nothing to prove about them.

- Suppose that the deformation is strong, so that $\mathrm{d} Y_{r}=\omega Y_{r}$ by Proposition 3.2. We prove that $\mathrm{d} C_{r}=0$ for any $r$. Indeed

$$
\begin{aligned}
\omega=\mathrm{d} Y_{r} Y_{r}^{-1} & =\mathrm{d} Y^{(0)}\left(Y^{(0)}\right)^{-1}+Y^{(0)} \mathrm{d} C_{r} C_{r}^{-1}\left(Y^{(0)}\right)^{-1} \\
& =\omega+Y^{(0)} \mathrm{d} C_{r} C_{r}^{-1}\left(Y^{(0)}\right)^{-1} \Longleftrightarrow \mathrm{d} C_{r}=0 .
\end{aligned}
$$

We prove that $d \mathbb{S}_{r}=0$. Invoking again Proposition 3.2, we have

$$
\omega_{j}=\frac{\partial Y_{r+1}}{\partial u_{j}} Y_{r+1}^{-1}=\frac{\partial Y_{r}}{\partial u_{j}} Y_{r}^{-1}+Y_{r} \frac{\partial \mathbb{S}_{r}}{\partial u_{j}} \mathbb{S}_{r}^{-1} Y_{r}^{-1}=\omega_{j}+Y_{r} \frac{\partial \mathbb{S}_{r}}{\partial u_{j}} \mathbb{S}_{r}^{-1} Y_{r}^{-1} \quad \Longleftrightarrow \quad \frac{\partial \mathbb{S}_{r}}{\partial u_{j}}=0 .
$$

- Conversely, we assume that $\mathrm{dS}_{0}=\mathrm{d} \mathbb{S}_{1}=\mathrm{d} B=\mathrm{d} C_{0}=\mathrm{d} L=0$, and that $D$ is constant. First, we see that $d \mathbb{S}_{r}=0$ and $\mathrm{d} C_{r}=0$ for any $r$, by formulae (3.2) and (3.3). By virtue of Proposition 3.2, it suffices to show that $\mathrm{d} Y_{r}=\omega Y_{r}$ for any $r$. By construction $\partial_{z} Y_{r}=\omega_{0}(z, u) Y_{r}$, which is the differential system (2.1), so it suffices to show that $\partial_{j} Y_{r}=\omega_{j} Y_{r}, j=1, \ldots, n$. Since $\mathrm{d} C_{0}=0$ and each $\mathrm{d} \mathbb{S}_{r}=0$, we have

$$
\mathrm{d} Y^{(0)}\left(Y^{(0)}\right)^{-1}=\mathrm{d} Y_{0} Y_{0}^{-1}=\cdots=\mathrm{d} Y_{r} Y_{r}^{-1}=\mathrm{d} Y_{r+1} Y_{r+1}^{-1}=\cdots \quad \forall r \in \mathbb{Z} .
$$

Moreover, $Y^{(0)}$ is a weak isomonodromic family, so that $\mathrm{d} Y^{(0)}=\omega Y^{(0)}$, where $\omega$ is $(2.20)$ with components (2.22), namely

$$
\omega_{j}(z, u)=z E_{j}+\left[F_{1}(u), E_{j}\right]+\mathcal{D}_{j}(u) .
$$

Thus, by (3.4), each $Y_{r}$ also satisfies

$$
\mathrm{d} Y_{r}=\omega Y_{r}, \quad \forall r .
$$

We need to prove that $\mathcal{D}_{j}(u)=0$. At $z=\infty$, using the fact that $d B=0$ and $\partial_{j} \Lambda=E_{j}$, we have for any $r \in \mathbb{Z}$

$$
\omega_{j}=\frac{\partial Y_{r}}{\partial u_{j}} Y_{r}^{-1}=\frac{\partial \widehat{Y}_{r}}{\partial u_{j}} \widehat{Y}_{r}^{-1}+z \widehat{Y}_{r} E_{j} \widehat{Y}_{r}^{-1}=z E_{j}+\left[F_{1}, E_{j}\right]+\mathcal{O}\left(z^{-1}\right) .
$$

The above $\mathcal{O}\left(z^{-1}\right)$ stands for an asymptotic expansion in $\mathcal{S}_{r}\left(\overline{\mathbb{D}^{\prime}}\right)$, given for any $r$ by the same series in $z^{-1}$. Since $\omega_{j}$ is single valued in $z$, then $\mathcal{O}\left(z^{-1}\right)$ is a convergent Taylor series at $z=\infty$. Thus, $\omega_{j}-z E_{j}-\left[F_{1}, E_{j}\right]$ is holomorphic both at $z=0$ and $z=\infty$, and it vanishes as $z \rightarrow \infty$. Hence, by Liouville theorem,

$$
\omega_{j}=z E_{j}+\left[F_{1}, E_{j}\right],
$$

with $\mathcal{D}_{j}=0$. The equality extends from $\mathbb{D}^{\prime}$ to $\mathbb{D}\left(u^{0}\right)$ by analiticity. 


\section{The non-generic case of coalescing eigenvalues of $\Lambda(u)$}

Having defined weak and strong isomonodromic deformations when the eigenvalues of $\Lambda$ are distinct and $A(u)$ is any, the next step towards non-generic isomonodromic deformations is to extended weak and strong deformations to the case when some eigenvalues of $\Lambda$ coalesce, namely when $u_{i}-u_{j} \rightarrow 0$ for some $i \neq j$. In this section, we give a "holomorphic" extension, summarised in Theorem 4.9 below, which constitutes one of the main results of [11].

Let $u^{C}=\left(u_{1}^{C}, \ldots, u_{n}^{C}\right) \in \mathbb{C}^{n}$ be a coalescence point (here, "C" stands for "coalescence"), namely

$$
u_{i}^{C}=u_{j}^{C} \quad \text { for some indexes } i \neq j \in\{1,2, \ldots, n\} .
$$

We consider a polydisc of radius $\epsilon>0$ centered at $u^{C}$ and denote it by $\mathcal{U}_{\epsilon}\left(u^{C}\right)$. It is to be noticed that there exists a coalescence locus in $\mathcal{U}_{\epsilon}\left(u^{C}\right)$, let it be denoted by $\Delta$, containing $u^{C}$ and defined by

$$
\Delta:=\mathcal{U}_{\epsilon}\left(u^{C}\right) \cap\left(\bigcup_{i \neq j}\left\{u_{i}-u_{j}=0\right\}\right) .
$$

In order to study the local theory at $u^{C}$, we assume that $\epsilon$ is small, so that $u^{C}$ is "the most" coalescent point. This means that if $k \neq l$ are indexes such that $u_{k}^{C}-u_{l}^{C} \neq 0$, then $\epsilon$ is sufficiently small to guarantee that $u_{k}-u_{l} \neq 0$ for every point of $\mathcal{U}_{\epsilon}\left(u^{C}\right)$.

We fix a half-line of direction $\arg z=\widetilde{\tau}$ in $\mathcal{R}$ which, now, does not coincide with any of the Stokes rays associated with $\Lambda\left(u^{C}\right)$, namely with the half lines in $\mathcal{R}$ specified by

$$
\operatorname{Re}\left(z\left(u_{k}^{C}-u_{l}^{C}\right)\right)=0, \quad \operatorname{Im}\left(z\left(u_{k}^{C}-u_{l}^{C}\right)\right)<0,
$$

for those $k, l$ such that $u_{k}^{C}-u_{l}^{C} \neq 0$. We call $\widetilde{\tau}$ an admissible direction at $u^{C}$. The choice of $\widetilde{\tau}$ determines a cell decomposition of $\mathcal{U}_{\epsilon}\left(u^{C}\right)$, which is based on two ingredients. One is $\Delta$ above. The other one is the so called "crossing locus". In order to describe it, observe that if $u \in \mathcal{U}_{\epsilon}\left(u^{C}\right)$ (points of $\Delta$ are not excluded) is such that $u_{i} \neq u_{j}$ for some $i \neq j$, then the (infinitely many in $\mathcal{R})$ Stokes rays

$$
\operatorname{Re}\left(z\left(u_{i}-u_{j}\right)\right)=0, \quad \operatorname{Im}\left(z\left(u_{i}-u_{j}\right)\right)<0,
$$

corresponding to $\left(u_{i}, u_{j}\right)$, are well defined. The crossing locus $X(\widetilde{\tau})$ is made of those points such that some Stokes rays "cross" the admissible rays $\{z \in \mathcal{R} \mid \arg z=\widetilde{\tau}+h \pi\}, h \in \mathbb{Z}$. Namely, $X(\widetilde{\tau})$ is made of points $u$ such that $\operatorname{Re}\left(e^{i \widetilde{\tau}}\left(u_{i}-u_{j}\right)\right)=0$ for some $u_{i} \neq u_{j}$. Precisely,

$$
X(\widetilde{\tau}):=\bigcup_{1 \leq i<j \leq n}\left\{u \in \mathcal{U}_{\epsilon}\left(u^{C}\right) \text { such that } u_{i} \neq u_{j} \text { and } \arg \left(u_{i}-u_{j}\right)=\frac{3 \pi}{2}-\widetilde{\tau} \bmod \pi\right\} .
$$

Let the "walls" be defined as

$$
W(\widetilde{\tau}):=\Delta \cup X(\widetilde{\tau})
$$

Following [11], every connected component of $\mathcal{U}_{\epsilon}\left(u^{C}\right) \backslash W(\widetilde{\tau})$ is called a $\widetilde{\tau}$-cell. We have proved in [11] that every $\widetilde{\tau}$-cell is a topological cell, so in particular it is simply connected (simple connectedness is important for the proof, given in [11], of Proposition 4.3 below).

The isomonodromy deformation theory can be extended, in a holomorphic way, to the case of coalescing eigenvalues when a certain vanishing condition holds for the entries of $A(u)$. 
Lemma 4.1. Consider the Pfaffian system (2.2)-(2.3), with $A(u)$ holomorphic on $\mathcal{U}_{\epsilon}\left(u^{C}\right)$. Let $\mathbb{D}\left(u^{0}\right)$ be compactly contained in a $\widetilde{\tau}$-cell. Assume that the Frobenius integrability holds on $\mathbb{D}\left(u^{0}\right)$ and that the $\omega_{j}(z, u)$ are holomorphic on $\mathbb{C} \times \mathbb{D}\left(u^{0}\right)$, with $z=\infty$ being at most a pole, so that Proposition 2.6 holds on $\mathbb{D}\left(u^{0}\right)$ and $(2.2)-(2.3)$ becomes system (2.20) with coefficients $(2.22)$ on $\mathbb{D}\left(u^{0}\right)$. Then, the following statements are true:

i) The coefficients $\omega_{j}(z, u)$ in (2.22) extend holomorphically on $\mathcal{U}_{\epsilon}\left(u^{C}\right)$ if and only if $\mathcal{D}_{j}(u)$ is holomorphic on $\mathcal{U}_{\epsilon}\left(u^{C}\right)$ and the following vanishing conditions hold in $\mathcal{U}_{\epsilon}\left(u^{C}\right)$ :

$$
A_{i j}(u)=\mathcal{O}\left(u_{i}-u_{j}\right) \quad \text { whenever } u_{i}-u_{j} \rightarrow 0 \text { at a point of } \Delta .
$$

In this case, the system (2.20), (2.22) is Frobenius integrable on the whole $\mathcal{U}_{\epsilon}\left(u^{C}\right)$.

ii) A fundamental matrix solution $Y(z, u)$ exists holomorphic on $\mathcal{R} \times \mathcal{U}_{\epsilon}\left(u^{C}\right)$ if and only if (4.1) holds.

Proof. The statement follows simply by recalling that, by Proposition 2.6, for $u \in \mathbb{D}\left(u^{0}\right)$ we have (2.22), namely

$$
\omega_{j}(z, u)=z E_{j}+\left(\frac{A_{a b}\left(\delta_{a j}-\delta_{b j}\right)}{u_{a}-u_{b}}\right)_{a, b=1}^{n}+\mathcal{D}_{j}(u),
$$

so that if $u_{i}-u_{j} \rightarrow 0$, the condition $A_{i j} \rightarrow 0$ guarantees analyticity on $\mathcal{U}_{\epsilon}\left(u^{C}\right)$. The integrability condition (2.4) then holds by analytic continuation from $\mathbb{D}\left(u^{0}\right)$ to $\mathcal{U}_{\epsilon}\left(u^{C}\right)$. The last statement concerning $Y(z, u)$ follows from the fact that if (4.1) holds, then the Pfaffian system is integrable and linear on $\mathcal{U}_{\epsilon}\left(u^{C}\right)$ with holomorphic (in $u$ ) coefficients. Conversely, if a fundamental matrix $Y(z, u)$ exists holomorphic on $\mathcal{R} \times \mathcal{U}_{\epsilon}\left(u^{C}\right)$, then $\omega=\mathrm{d} Y Y^{-1}$ has coefficients holomorphic in $u$ on $\mathcal{U}_{\epsilon}\left(u^{C}\right)$, which implies (4.1).

Under the assumptions of Lemma 4.1, then Propositions 2.3 and 2.4 hold with the replacements $u^{0} \mapsto u^{C}$ and $\mathbb{D}\left(u^{0}\right) \mapsto \mathcal{U}_{\epsilon}\left(u^{C}\right)$, if and only if $A_{i j}(u)=\mathcal{O}\left(u_{i}-u_{j}\right)$ for $u_{i}-u_{j} \rightarrow 0$ at $\Delta$. Namely

Corollary 4.2. Under the assumptions of Lemma 4.1, $A(u)$ is holomorphically similar to a constant Jordan form $J$ on $\mathcal{U}_{\epsilon}\left(u^{C}\right)$, and there exists an isomonodromic family of fundamental solutions $Y^{(0)}(z, u)$ in Levelt form (2.11)-(2.12), holomorphic on $\mathcal{R} \times \mathcal{U}_{\epsilon}\left(u^{C}\right)$, with constant $L$ and $D$, if and only if $A_{i j}(u)=\mathcal{O}\left(u_{i}-u_{j}\right)$ for $u_{i}-u_{j} \rightarrow 0$ at $\Delta$.

We turn to the fundamental matrices $Y_{r}(z, u)$ in $(2.17)-(2.18)$. Notice that $\widetilde{\tau}$ is admissible at any $u \in \mathbb{D}\left(u^{0}\right)$, because $\mathbb{D}\left(u^{0}\right)$ is assumed to be compactly contained in a $\widetilde{\tau}$-cell. Then, the matrices $Y_{r}(z, u)$ are well defined and holomorphic on a small $\mathbb{D}^{\prime} \subset \mathbb{D}\left(u^{0}\right)$, as proved by Sibuya, and all the results described in Section 2 apply on $\mathbb{D}^{\prime}$. Moreover, by item (F) of Proposition 2.6, $\mathbb{D}^{\prime}$ is extended to $\mathbb{D}\left(u^{0}\right)$, so that the fundamental matrices $Y_{r}(z, u)$ are holomprphic on $\mathcal{R} \times \mathbb{D}\left(u^{0}\right)$. Complications arise if we want to study the $u$-analytic continuation of the matrices $Y_{r}(z, u)$ on the whole $\mathcal{U}_{\epsilon}\left(u^{C}\right)$, and their asymptotic behaviour.

The problem can indeed be studied also in the non-isomonodromic case, when the $Y_{r}(z, u)$ 's are defined only for $u \in \mathbb{D}^{\prime}$. When $u$ moves outside $\mathbb{D}^{\prime}$, Sibuya's local result concerning analyticity in $u$ does no longer apply. This issue was analyszed in [11], for a system (2.1) without any assumption that it be isomonodromic, namely without assuming that there is a Pfaffian system behind (2.1). The following holds (indeed, for the more general system (4.7) below).

Proposition 4.3 ([11]). Let $A(u)$ be holomorphic on $\mathcal{U}_{\epsilon}\left(u^{C}\right)$. For any $z \in \mathcal{R}$, the fundamental matrices $Y_{r}(z, u), r \in \mathbb{Z}$, of a differential system (2.1) or (4.7), not necessarily isomonodromic, defined on $\mathbb{D}^{\prime} \subset \mathbb{D}\left(u^{0}\right)$ (on $\mathbb{D}\left(u^{0}\right)$, in the isomonodromic case) can be analytically continued 
w.r.t. $u$ on the whole $\widetilde{\tau}$-cell containing $u^{0}$, maintaining the asymptotics (2.18). The asymptotics is uniform in any compact subset $K$ of the cell, for $z \rightarrow \infty$ in the following sector

$$
\mathcal{S}_{r}(K):=\bigcap_{u \in K} \mathcal{S}_{r}(u)
$$

Moreover, the analytic continuation - maintaining for each $u$ the asymptotics in $\mathcal{S}_{r}(u)$ - exists along any curve slightly beyond the boundary of the cell, if the curve crosses the boundary at a point corresponding to just one Stokes ray crossing $\arg z=\widetilde{\tau}$ (simple crossing).

We refer to [11] for the proof. Here we notice that in the strong isomonodromic case of system (2.1), it is easy to show the existence of the analytic continuation claimed in Proposition 4.3. Indeed, if $A(u)$ is holomorphic on $\mathcal{U}_{\epsilon}\left(u^{C}\right)$, the analytic continuation exists beyond the $\widetilde{\tau}$-cell containing $u^{0}$, over any simply connected subset of $\mathcal{U}_{\epsilon}\left(u^{C}\right) \backslash \Delta$ containing $u^{0}$, because

$$
\omega_{j}(z, u)=z E_{j}+\left(\frac{A_{a b}\left(\delta_{a j}-\delta_{b j}\right)}{u_{a}-u_{b}}\right)_{a, b=1}^{n}
$$

is holomorphic on $\mathcal{U}_{\epsilon}\left(u^{C}\right) \backslash \Delta$. Therefore, since $\mathrm{d} Y_{r}=\omega Y_{r}$, then $Y_{r}$ enjoys the properties of the solutions of a linear Pfaffian system with holomorphic coefficients away form $\Delta$. However, the non-isomonodromic case (system (2.1) or (4.7)) requires a more sophisticated extension of Sibuya's results. Moreover, the proof that the analytic continuation of $Y_{r}(z, u)$ maintains the asymptotic behaviour $Y_{F}(z, u)$ on $\mathcal{S}_{r}(K)$ is non trivial, both in the non-isomonodromic and isomonodromic cases. See [11, Chapters 12 and 13] for details.

Having established in Proposition 4.3 the analytic continuation and the asymptotics on a $\widetilde{\tau}$ cell, we have to study what happens if $u$ moves outside the cell along a curve crossing $W(\widetilde{\tau})$. We face further problems:

i) When the crossing occurs at a point of $\Delta$, the coefficients $F_{k}(u)$ in $Y_{F}(z, u)$ have poles for $u_{i}-u_{j}=0$, because (see [11])

$$
\begin{aligned}
& \left(F_{k}\right)_{i j}=\frac{1}{u_{j}-u_{i}}\left\{\left(A_{i i}-A_{j j}+k-1\right)\left(F_{k-1}\right)_{i j}+\sum_{p \neq i} A_{i p}\left(F_{k-1}\right)_{p j}\right\}, \quad i \neq j ; \\
& k\left(F_{k}\right)_{i i}=-\sum_{j \neq i} A_{i j}\left(F_{k}\right)_{j i} .
\end{aligned}
$$

ii) An actual solution $Y_{r}(z, u)$ is in general multivalued for loops around $u_{i}-u_{j}=0$ and diverges as $u_{i}-u_{j} \rightarrow 0$ along any direction (provided we are in a case when $Y_{r}(z, u)$ can be extended analytically along any curve outside the cell, as it certainly happens for strong isomonodromic deformations, as discussed in the comments after Proposition 4.3). The reader can find some explicit examples in the papers [15] and [22], respectively based on talks at workshops in CRM, Pisa (February 13-17, 2017) and at the University of Alcalá (September 4-8, 2017).

iii) The asymptotic behaviour of $Y_{r}(z, u)$ in sectors $\mathcal{S}_{r}(K)$ does no longer hold when $u$ is outside the cell containing $u^{0}$, because some Stokes rays cross the admissible direction $\widetilde{\tau}$ (again, provided that $Y_{r}(z, u)$ can be extended analytically to some simply connected subset of $\mathcal{U}_{\epsilon}\left(u^{C}\right) \backslash \Delta$ containing the cell, as it happens in the case of strong isomonodromic deformations).

The issue above can be solved in the strong isomonodromic case when the vanishing conditions (4.1) hold. Let the deformation be strongly isomonodromic in $\mathbb{D}\left(u^{0}\right)$ compactly contained in a $\widetilde{\tau}$-cell, so that

$$
\mathrm{d} Y_{r}=\omega Y_{r}
$$


on $\mathbb{D}\left(u^{0}\right)$, with $\omega$ as in (3.1). Since (4.4) is linear, and its coefficients are holomorphic on the whole $\mathcal{U}_{\epsilon}\left(u^{C}\right)$ if and only if conditions (4.1) hold, then the following proposition holds.

Proposition 4.4. In case of strong isomonodromy deformations, the fundamental matrices $Y_{r}(z, u)$ extend to holomorphic functions on $\mathcal{R} \times \mathcal{U}_{\epsilon}\left(u^{C}\right)$ if and only if the vanishing conditions (4.1) hold.

As far as the formal fundamental matrix $Y_{F}(z, u)$ is concerned, we have the following

Proposition 4.5. Let $A(u)$ be holomorphic on $\mathcal{U}_{\epsilon}\left(u^{C}\right)$ and assume that the vanishing conditions (4.1) hold in $\mathcal{U}_{\epsilon}\left(u^{C}\right)$. Then, in case of strong isomonodromic deformations, the coefficients $F_{k}(u), k \geq 1$, of the formal solution $Y_{F}(z, u)$ are holomorphic on $\mathcal{U}_{\epsilon}\left(u^{C}\right)$.

Proof. The coefficients $F_{k}(u)$ are computed recursively from (2.1). This standard computation yields coefficients depending rationally on $A(u)$ and on the differences $\left(u_{i}-u_{j}\right)$, which appear in the denominators, as in (4.2). In particular (2.21) holds:

$$
\left(F_{1}\right)_{i j}(u)=\frac{A_{i j}(u)}{u_{j}-u_{i}}, \quad i \neq j, \quad\left(F_{1}\right)_{i i}(u)=-\sum_{j \neq i} A_{i j}(u)\left(F_{1}\right)_{j i}(u) .
$$

Thus, if (4.1) holds, $F_{1}(u)$ is holomorphic in $\mathcal{U}_{\epsilon}\left(u^{C}\right)$. By Proposition 4.3, the asymptotic expansion is uniform in compact subsets of a cell, so we can substitute it into $\partial_{i} Y=\omega_{i} Y$ (with $\omega_{i}$ as in (3.1)), compare coefficients of $z^{-l}$ and find

$$
\left[F_{l+1}(u), E_{i}\right]=\left[F_{1}(u), E_{i}\right] F_{l}(u)-\partial_{i} F_{l}(u), \quad l \geq 1,
$$

with

$$
\left[F_{l+1}(u), E_{i}\right]=\left(\begin{array}{ccccc}
0 & & \left(F_{l+1}\right)_{1 i} & & 0 \\
& & \vdots & & \\
-\left(F_{l+1}\right)_{i 1} & \cdots & 0 & \cdots & -\left(F_{l+1}\right)_{i n} \\
& & \vdots & & \\
0 & & \left(F_{l+1}\right)_{n i} &
\end{array}\right) .
$$

Moreover, $\operatorname{diag}\left(F_{l+1}\right)$ is determined by

$$
l\left(F_{l+1}\right)_{i i}(u)=-\sum_{j \neq i} A_{i j}(u)\left(F_{l}\right)_{j i}(u) .
$$

Therefore, (4.5)-(4.6) recursively determines $F_{l+1}$ as a function of $F_{l}, F_{l-1}, \ldots, F_{1}$. Since $F_{1}$ is holomorphic when conditions (4.1) hold, by induction all the $F_{l+1}(u)$ are holomorphic.

Remark 4.6. Proposition 4.5 holds only in the strong isomonodromic case. In the nonisomonodromic case the vanishing conditions (4.1) only guarantee that $F_{1}(u)$ is holomorphic. In order for $F_{1}, F_{2}, \ldots, F_{l}$ to be holomorphic up to a certain $l$, also the following quantities

$$
\left(A_{i i}-A_{j j}+k-1\right)\left(F_{k-1}\right)_{i j}+\sum_{p \neq i} A_{i p}\left(F_{k-1}\right)_{p j}, \quad 2 \leq k \leq l,
$$

must vanish when $u_{i}-u_{j} \rightarrow 0$. The above, which follow from (4.2)-(4.3), are conditions on the entries of $A$, since the $F_{k}$ are determined by $A$. 
It remains to check what happens to the asymptotic behaviour of the matrices $Y_{r}(z, u)$ outside the cell. This requires a certain amount of non-trivial work, which we have done in [11] for a system of the form

$$
\frac{\mathrm{d} Y}{\mathrm{~d} z}=\left(\Lambda+\frac{A(u)}{z}+\sum_{j=2}^{\infty} \frac{A_{j}(u)}{z^{j}}\right) Y, \quad u \in \mathcal{U}_{\epsilon}\left(u^{C}\right),
$$

without assuming that the system is isomonodromic. The series above is assumed to converge at $z=\infty$ with holomorphic matrix coefficients $A_{j}(u)$ on $\mathcal{U}_{\epsilon}\left(u^{C}\right)$. The asymptotic theory at $z=\infty$ for (4.7), with $u \in \mathbb{D}^{\prime}$ sufficiently small contained in a $\widetilde{\tau}$-cell, is the same as for (2.1). Namely, there is a unique formal solution

$$
Y_{F}(z, u)=F(z, u) z^{B(u)} e^{z \Lambda}, \quad B(u)=\operatorname{diag}(A(u)), \quad F(z, u)=I+\sum_{k=1}^{\infty} F_{k}(u) z^{-k},
$$

and unique actual solutions $Y_{r}(z, u)$.

In order to proceed, we need to take $\epsilon$ sufficiently small, as follows. Consider the sub-class of Stokes rays associated with the pairs of eigenvalues $u_{i}$ and $u_{j}$, with label $i, j$ corresponding to components of $u^{C}$ satisfying $u_{i}^{C} \neq u_{j}^{C}$. If $\epsilon$ is small enough ${ }^{5}$, these rays do not cross any admissible direction $\widetilde{\tau}+h \pi$ when $u$ varies in $\mathcal{U}_{\epsilon}\left(u^{C}\right)$. We define a sector $\widehat{\mathcal{S}}_{r}(u) \subset \mathcal{R}$, which contains the "half-plane" $\widetilde{\tau}+(r-2) \pi<\arg z<\widetilde{\tau}+(r-1) \pi$ and extends up to the nearest Stokes rays lying outside the "half-plane" in the sub-class above (notice that $\mathcal{S}_{r}(u) \subset \widehat{\mathcal{S}}_{r}(u)$ ). Then, we define

$$
\widehat{\mathcal{S}}_{r}:=\bigcap_{u \in \mathcal{U}_{\epsilon}\left(u^{C}\right)} \widehat{\mathcal{S}}_{r}(u)
$$

The sectors $\widehat{\mathcal{S}}_{r}$ have angular opening greater than $\pi$. The following theorem has been proved in [11]. It requires a non-trivial amount of work, which we necessarily skip here.

Theorem 4.7 ([11]). Consider the differential system (4.7), with coefficients $A(u), A_{j}(u)$ holomorphic on $\mathcal{U}_{\epsilon}\left(u^{C}\right)$, where $\epsilon$ is specified as above. Assume that all the $F_{k}(u)$ are holomorphic on $\mathcal{U}_{\epsilon}\left(u^{C}\right)$. Moreover, assume that the fundamental matrices

$$
Y_{r}(z, u)=\widehat{Y}_{r}(z, u) z^{B(u)} e^{z \Lambda},
$$

which are holomorphic on a $\widetilde{\tau}$-cell by Proposition 4.3, admit analytic continuation on the whole $\mathcal{U}_{\epsilon}\left(u^{C}\right)$ as single valued holomorphic functions of $u$, for any $r \in \mathbb{Z}$ and $z$ fixed. Then the following results hold.

- The asymptotic representation of $Y_{r}(z, u)$ in terms of the formal matrix

$$
Y_{F}(z, u)=F(z, u) z^{B(u)} e^{z \Lambda},
$$

extends beyond the $\widetilde{\tau}$-cell, namely

$$
\widehat{Y}_{r}(z, u) \sim F(z, u)=I+\sum_{k=1}^{\infty} F_{k}(u) z^{-k}, \quad z \rightarrow \infty \text { in } \widehat{\mathcal{S}}_{r},
$$

uniformly in every compact subset of $\mathcal{U}_{\epsilon_{1}}\left(u^{C}\right)$ for every $\epsilon_{1}<\epsilon$. Moreover, for any $r \in \mathbb{Z}$ the Stokes matrix $\mathbb{S}_{r}(u)$ relating $Y_{r}(z, u)$ and $Y_{r+1}(z, u)$ satisfies

$$
\left(\mathbb{S}_{r}\right)_{i j}=\left(\mathbb{S}_{r}\right)_{j i}=0 \quad \text { for } i, j \text { such that } u_{i}^{C}=u_{j}^{C} \text {. }
$$

\footnotetext{
${ }^{5}$ It suffices to take $\epsilon$ less than the minimum over $i$ and $j$, such that $u_{i}^{C} \neq u_{j}^{C}$, of the distances between the two parallel lines in the complex plane, one passing through $u_{i}^{C}$ and one through $u_{j}^{C}$, with direction $3 \pi / 2-\widetilde{\tau}(\bmod \pi$, or mor $2 \pi$, which is the same).
} 
- The system (4.7) at fixed $u=u^{C}$, namely

$$
\frac{\mathrm{d} Y}{\mathrm{~d} z}=\left(\Lambda\left(u^{C}\right)+\frac{A\left(u^{C}\right)}{z}+\sum_{j=2}^{\infty} \frac{A_{j}\left(u^{C}\right)}{z^{j}}\right) Y,
$$

has formal solutions with the following structure ${ }^{6}$

$$
\stackrel{\circ}{Y}_{F}(z)=\stackrel{\circ}{F}(z) z^{B\left(u^{C}\right)} e^{z \Lambda\left(u^{C}\right)},
$$

where $\stackrel{\circ}{F}(z)=I+\sum_{f=1}^{\infty} \stackrel{\circ}{F}_{K} z^{-k}$ is a formal series and $B\left(u^{C}\right)=\operatorname{diag}\left(A_{11}\left(u^{C}\right), \ldots, A_{n n}\left(u^{C}\right)\right)$.

- The solution $\dot{Y}_{F}(z)$ is unique if and only if the diagonal entries of $A\left(u^{C}\right)$ do not differ by non-zero integers; otherwise, there is a family of formal solutions $\dot{Y}_{F}(z)$.

- For any fixed formal solution $\stackrel{\circ}{Y}_{F}(z)$ of (4.8), there are unique actual solutions

$$
\dot{\circ}_{r}(z)=\stackrel{\widehat{\hat{Y}}}{r}_{r}(z) z^{B\left(u^{C}\right)} e^{z \Lambda\left(u^{C}\right)}, \quad r \in \mathbb{Z}
$$

such that

$$
\stackrel{\circ}{Y}_{r}(z) \sim \stackrel{\circ}{F}(z) \quad \text { for } z \rightarrow \infty \text { in } \widehat{\mathcal{S}}_{r}\left(u^{C}\right) .
$$

- In particular, there exists a formal solution $\stackrel{\circ}{Y}_{F}(z)$ of (4.8) satisfying

$$
\stackrel{\circ}{Y}_{F}(z)=Y_{F}\left(z, u^{C}\right) .
$$

The corresponding unique actual solutions $\stackrel{\circ}{Y}_{r}(z)$ satisfy

$$
\stackrel{\circ}{Y}_{r}(z)=\lim _{u \rightarrow u_{c}} Y_{r}(z, u) \equiv Y_{r}\left(z, u^{C}\right) .
$$

Let $\stackrel{\circ}{\mathbb{S}}_{r}$ be the Stokes matrices of the above solutions $\stackrel{\circ}{Y}_{r}(z)$. Then,

$$
\lim _{u \rightarrow u_{c}} \mathbb{S}_{r}(u)=\stackrel{\circ}{S}_{r}
$$

Remark 4.8. We use the notation $\stackrel{\circ}{Y}, \stackrel{\circ}{F}, \stackrel{\circ}{\mathbb{S}}$, etc., for objects $Y, F, \mathbb{S}$, etc, relative to the system at $u=u^{C}$, such as (4.8) above and (4.10) below.

Theorem 4.7 is at the core of the validity of one of the main results of [11], namely Theorem 4.9 below. Indeed, by Propositions 4.4 and 4.5, the assumptions of Theorem 4.7 hold in the strong isomonodromic case if the vanishing conditions (4.1) hold. This yields the following

Theorem 4.9 ([11]). Let $A(u)$ be holomorphic on $\mathcal{U}_{\epsilon}\left(u^{C}\right)$ and $\epsilon$ small as specified above. If the system

$$
\partial_{z} Y=\left(\Lambda+\frac{A(u)}{z}\right) Y
$$

is strongly isomonodromic on a polydisk interior to a $\widetilde{\tau}$-cell and the vanishing conditions (4.1) hold, then:

\footnotetext{
${ }^{6}$ Without the assumptions of the theorem, the formal solutions have a more complicated structure. See [3, 4] for a general theory, and [11] for the specific case here studied.
} 
a) the coefficients of the unique formal solution $Y_{F}(z, u)=F(z, u) z^{B} e^{z \Lambda}$ are holomorphic on $\mathcal{U}_{\epsilon}\left(u^{C}\right)$, and the corresponding actual solutions $Y_{r}(z, u)$ extend holomorphically on $\mathcal{R} \times \mathcal{U}_{\epsilon}\left(u^{C}\right)$, maintaining the asymptotics

$$
\widehat{Y}_{r}(z, u) \sim F(z, u) \quad \text { for } z \rightarrow \infty \text { in } \widehat{\mathcal{S}}_{r},
$$

uniformly in every compact subset of $\mathcal{U}_{\epsilon_{1}}\left(u^{C}\right)\left(\forall \epsilon_{1}<\epsilon\right)$.

b) For any $r$, the essential monodromy data $\mathbb{S}_{r}, \mathbb{S}_{r+1}, B, L, D$ and $C_{r}$ are constant on the whole $\mathcal{U}_{\epsilon}\left(u^{C}\right)$ and satisfy

$$
\left(\mathbb{S}_{r}\right)_{i j}=\left(\mathbb{S}_{r+1}\right)_{i j}=\left(\mathbb{S}_{r}\right)_{j i}=\left(\mathbb{S}_{r+1}\right)_{j i}=0 \quad \text { for } i, j \text { such that } u_{i}^{C}=u_{j}^{C} .
$$

c) They coincide with a set of essential monodromy data of

$$
\partial_{z} Y=\left(\Lambda\left(u^{C}\right)+\frac{A\left(u^{C}\right)}{z}\right) Y .
$$

in the following way. Take the formal solution $\stackrel{\circ}{Y}_{F}(z)$ of (4.10) satisfying

$$
\stackrel{\circ}{Y}_{F}(z)=Y_{F}\left(z, u^{C}\right)
$$

and the associated actual solutions $\stackrel{\circ}{Y}_{r}(z)$, with Stokes matrices $\stackrel{\circ}{\mathbb{S}}_{r}$. Then, choose a fundamental matrix solution of (4.10) in Levelt form

$$
\stackrel{\circ}{Y}^{(0)}(z)=\stackrel{\circ}{\hat{Y}}(z) z^{D} z^{\stackrel{\circ}{L}}
$$

and take the corresponding connection matrix $\stackrel{\circ}{r}_{r}$ such that

$$
\stackrel{\circ}{Y}_{r}(z)=\stackrel{\circ}{Y}^{(0)}(z) \stackrel{\circ}{C}_{r}
$$

Then, there exists a fundamental matrix $Y^{(0)}(z, u)$ of (4.9) in Levelt form such that the essential monodromy data of (4.9) on the whole $\mathcal{U}_{\epsilon}\left(u^{C}\right)$ are

$$
B, \quad \mathbb{S}_{r}=\stackrel{\circ}{\mathbb{S}}_{r}, \quad \mathbb{S}_{r+1}=\stackrel{\circ}{\mathbb{S}}_{r+1}, \quad C_{r}=\stackrel{\circ}{C}_{r}, \quad L=\stackrel{\circ}{L}, \quad D .
$$

d) If the diagonal entries of $A\left(u^{c}\right)$ do not differ by non-zero integers, then there is a unique formal solution $\stackrel{\circ}{Y}_{F}(z)$ which necessarily satisfies $\stackrel{\circ}{Y}_{F}(z)=Y_{F}\left(z, u^{C}\right)$.

Proof. Points a), b), c) follow from Propositions 4.4 and 4.5, Theorem 4.7 and Corollary 4.2. We only need to justify that given a fundamental matrix $\stackrel{\circ}{Y}^{(0)}(z)$ of $(4.10)$ in Levelt form (4.11), there exists a fundamental matrix $Y^{(0)}(z, u)$ of $\mathrm{d} Y=\omega(z, u) Y$ in Levelt form (namely an isomonodromic fundamental matrix of (4.9)), such that

$$
Y^{(0)}\left(z, u^{C}\right)=\stackrel{\circ}{Y}^{(0)}(z) .
$$

To this end, recall the proof of Proposition 2.4, with the replacements $u \mapsto u^{C}$ and $\mathbb{D}\left(u^{0}\right) \mapsto$ $\mathcal{U}_{\epsilon}\left(u^{0}\right)$ (this becomes the proof of Corollary 4.2). The gauge transformation (2.13)

$$
Y=V(z, u) \tilde{Y}, \quad V\left(z, u^{C}\right)=I,
$$

transforms the Pfaffian system to a differential system (2.14) in variable $z$ only, with $u^{0}$ replaced by $u^{C}$, namely

$$
\mathrm{d} \widetilde{Y}=\left[\left(\frac{A\left(u^{C}\right)}{z}+\Lambda\left(u^{C}\right)\right) \mathrm{d} z\right] \tilde{Y}, \quad \mathrm{~d} \equiv \frac{\partial}{\partial z} \mathrm{~d} z .
$$


This is exactly (4.10). A fundamental solution $\stackrel{\circ}{Y}^{(0)}(z)$ in Levelt form (4.11) is actually a solution (2.15), namely

$$
\stackrel{\circ}{Y}^{(0)}(z)=W(z) z^{D} z^{\stackrel{\circ}{L},} \quad W(z)=W_{0}+\sum_{\ell=1}^{\infty} W_{\ell} z^{\ell}, \quad \operatorname{det}\left(W_{0}\right) \neq 0 .
$$

Thus, we conclude from (2.13) that

$$
Y^{(0)}(z, u)=V(z, u) \stackrel{\circ}{Y}^{(0)}(z), \quad Y^{(0)}\left(z, u^{C}\right)=\stackrel{\circ}{Y}^{(0)}(z) .
$$

Point d) follows from the recursive computation of the coefficients $\stackrel{\circ}{F}_{k}$ in

$$
\stackrel{\circ}{Y}_{F}(z)=\left(I+\sum_{k=1}^{\infty} \stackrel{\circ}{F}_{k} z^{-k}\right) z^{B\left(u^{C}\right)} e^{z \Lambda\left(u^{C}\right)} .
$$

For $1 \leq \ell \neq s \leq n$ such that $u_{\ell}^{C}=u_{s}^{C}$, one finds at step $k$ that (recall that $\operatorname{diag}(A(u))=B$ is constant)

$$
\left(A_{\ell \ell}-A_{s s}+k\right)\left(\stackrel{\circ}{F}_{k}\right)_{\ell s}=\text { known expression from previous steps. }
$$

This implies that if $A_{\ell \ell}-A_{s s}+k$ is different from zero for every $k \geq 1$, then all the $\stackrel{\circ}{F}_{k}$ are uniquely recursively determined. For the detailed formulae of the recursion, see [11, Section 4].

In presence of the vanishing conditions (4.1), Theorem 4.9 assures that in order to find the monodromy data of the differential system (4.9) on $\mathcal{U}_{\epsilon}\left(u^{C}\right)$, we just need to find the monodromy data associated with the fundamental matrices $\stackrel{\circ}{Y}_{r}(z), \stackrel{\circ}{Y}_{r+1}(z)$ of $(4.10)$ asymptotic to $\stackrel{\circ}{Y}_{F}(z)=$ $Y_{F}\left(z, u^{C}\right)$ in $\widehat{\mathcal{S}}_{r}\left(u^{C}\right)$, and with a solution $\stackrel{\circ}{Y}^{(0)}(z)=\stackrel{\widehat{Y}}{Y}(z) z^{D} z^{\stackrel{\circ}{L}}$ of $(4.10)$ in Levelt form.

The computation of monodromy data of (4.9) (namely (2.1)) is highly transcendental, so that even if $A(u)$ is completely known in a neighbourhood of $u^{C}$, in general it cannot be done at a generic $u \in \mathcal{U}_{\epsilon}\left(u^{C}\right)$. On the other hand, it may happen that it can be explicitly done for (4.10), because the system simplifies at the coalescence point, thanks to the null entries

$$
A_{i j}\left(u^{C}\right)=0 \quad \text { whenever } \quad u_{i}^{C}=u_{j}^{C} .
$$

By Theorem 4.9, the result so obtained yields the constant monodromy data of (4.9) in a neighbourhood of $u^{C}$. An example of this has been given in [11] concerning monodromy data of a Painlevé equation, and in [12] for the monodromy data of the Frobenius manifold associated with the reflection group $A_{3}$.

Theorem 4.9 allows to compute monodromy data in a neighbourhood of a coalescence point also in cases when we miss some information about the system away form $u^{C}$. Indeed, suppose that the system (4.9) is known only at a coalescence point $u^{C}$; namely, we only know the explicit form of $A\left(u^{C}\right)$. Moreover, suppose that for some theoretical reason it is known that in a neighbourhood of $u^{C}$ the unknown $A(u)$ must satisfy the vanishing conditions (4.1). Then, Theorem 4.9 yields the essential monodromy data in a whole neighbourhood of $u^{C}$ without knowing explicitly $A(u)$ for $u \neq u^{C}$. This approach has been used in [12] to compute the monodromy data of the quantum cohomology of the Grassmannian $\operatorname{Gr}(2,4)$, and to prove in a completely explicit way (i.e., by computations of the numerical values of the data) a conjecture $[12,13,17,20]$ relating them to exceptional collections in derived categories of coherent sheaves on $\operatorname{Gr}(2,4)$ (for a shorter summary, see also [14], which is based on a talk at CRM, Pisa, February 13-17, 2017). Notice that the quantum cohomology of almost all Grassmannians is characterised by a coalescence phenomenon [10] and that Theorem 4.9 applies, due to the semisimplicity of these quantum cohomologies. This fact justifies the computation of the monodromy data for Grassmannians starting from a coalescence point. 
Remark 4.10. When applying Theorem 4.9 as explained above, among the possible formal solutions $\stackrel{\circ}{Y}_{F}(z)$ of (4.10), we need precisely the one satisfying $\stackrel{\circ}{Y}_{F}(z)=Y_{F}\left(z, u^{C}\right)$. The fact that we do not have this information without knowing already $Y_{F}(z, u)$ may constitute a difficulty. Fortunately, the problem does not arise when the diagonal entries of $A\left(u^{C}\right)$ do not differ by nonzero integers, because in this case (4.10) only has the unique formal solution $\stackrel{\circ}{F}_{F}(z)=Y\left(z, u^{C}\right)$. In applications to Frobenius manifolds, discussed in $[11,12,13,14]$, we are in this good situation. Notice that the Stokes matrices are completely determined by (4.10) in this case. ${ }^{7}$

Remark 4.11. For a system analogous to (2.1), associated with a semisimple Frobenius manifold, where $A(u)$ is skew symmetric, a synthetic proof is given in [20] that a fundamental matrix solution asymptotic to the formal solution in a sector of central opening angle $\pi+\varepsilon$ (the analogous to our $\left.Y_{r}(z, u) \sim Y_{F}(z, u)\right)$ is holomorphic in a small neighbourhood of a coalescence point $u^{C}$. This result, in case of Frobenius manifolds, is analogous of the first point of Theorem 4.9. The proof in [20] is based on the Laplace transformation of the irregular system into an isomonodromic Fuchsian system, whose associated Pfaffian system is of Fuchsian type and has the good analyticity properties discussed in [34].

\section{A Weak and strong isomonodromic deformations of Fuchsian systems}

The difference between weak and strong isomonodromic deformations naturally arises in case of Fuchsian systems. This is synonymous of Schlesinger and non-Schlesinger deformations studied by Bolibruch.

Up to a Möbius transformation, we can assume that $z=\infty$ is non singular. Accordingly, we consider the $u$-family of $n \times n$ Fuschsian systems

$$
\frac{\mathrm{d} Y}{\mathrm{~d} z}=\sum_{i=1}^{N} \frac{A_{i}(u)}{z-u_{i}} Y, \quad \sum_{i=1}^{N} A_{i}(u)=0,
$$

depending holomorphically on the parameter $u=\left(u_{1}, \ldots, u_{N}\right)$ in a polydisc $\mathbb{D}\left(u^{0}\right)$ with center $u^{0}=\left(u_{1}^{0}, \ldots, u_{N}^{0}\right)$, contained in $\mathbb{C}^{N} \backslash \bigcup_{i \neq j}\left\{\left(u_{i}-u_{j}\right)=0\right\}$. We take $(z, u)$ in

$$
\mathcal{E}:=\left(\mathbb{P}^{1} \times \mathbb{D}\left(u^{0}\right)\right) \backslash \bigcup_{i=1}^{N}\left\{\left(z-u_{i}\right)=0\right\} .
$$

Notice that $\mathbb{D}\left(u^{0}\right)=\mathbb{D}_{1} \times \cdots \times \mathbb{D}_{N}$, where $\mathbb{D}_{i}$ is a disk centered at $u_{i}^{0}$ and $\overline{\mathbb{D}}_{i} \cap \overline{\mathbb{D}}_{j}=\emptyset$. Thus, the fundamental group of $\mathbb{P}^{1} \backslash\left\{u_{1}, \ldots, u_{N}\right\}$ can be defined in a $u$-independent way. ${ }^{8}$

Definition A.1. The family of systems (A.1) is weakly isomonodromic if there exists a family of fundamental matrix solutions $Y(z, u)$ having the same monodromy matrices for all $u \in \mathbb{D}\left(u_{0}\right)$. This family $Y(z, u)$ is called a weak isomonodromic family of fundamental matrices.

It follows from the theorem on analytic dependence on parameters that there exists a family of fundamental matrices $\widetilde{Y}(z, u)$ which is analytic in $(z, u)$ on the universal covering of $\mathcal{E}$, but which is not necessarily isomonodromic. Namely, $\widetilde{Y}(z, u)=Y(z, u) C(u)$ for some connection matrix $C(u)$. If $M_{1}, \ldots, M_{N}$ are the monodromy matrices of $Y(z, u)$ w.r.t. a basis of

\footnotetext{
${ }^{7}$ Since the choice of a solution in Levelt form is not unique, there may be a freedom in the data $C_{r}, D, L$. Only the Stokes matrices are uniquely determined.

${ }^{8}$ In other words, $\mathcal{E}=\mathbb{P}^{1} \times \mathbb{D}\left(u^{0}\right) \backslash \bigcup_{i=1}^{N}\left\{\left(z-u_{i}\right)=0\right\}$ can be retracted to $\mathbb{P}^{1} \backslash\left\{u_{1}^{0}, \ldots, u_{N}^{0}\right\}$, and $\pi_{1}\left(\mathcal{E} ;\left(z_{0}, u^{0}\right)\right)$ is isomorphic to $\pi_{1}\left(\mathbb{P}^{1} \backslash\left\{u_{1}^{0}, \ldots, u_{N}^{0}\right\} ; z_{0}\right)$.
} 
$\pi_{1}\left(\mathbb{P}^{1} \backslash\left\{u_{1}^{0}, \ldots, u_{N}^{0}\right\} ; z_{0}\right)$, then $\tilde{Y}(z, u)$ has non-constant matrices $M_{j}(u)=C(u)^{-1} M_{j} C(u)$. For a Fuchsian isomonodromic system, it can be proved that there exists an isomonodromic $Y(z, u)$ such that $C(u)$ is holomorphic. Namely:

Proposition A.2 ([8,9]). If (A.1) is weakly isomonodromic, then there exists an isomonodromic family of fundamental matrices $Y(z, u)$ which is holomorphic in $z$ and $u$ on the universal covering of $\mathcal{E}$.

The proof makes use of holomorphic bundles, and we refer to $[8,9]$.

Theorem A.3 ([8, 9]). The u-family of Fuschsian systems (A.1) is weakly isomonodromic if and only if there exists a matrix valued 1 -form $\omega$, holomorphic on $\mathcal{E}$, such that

1) for any fixed $u \in \mathbb{D}\left(u^{0}\right)$ we have

$$
\left.\omega\right|_{u}=\sum_{i=1}^{N} \frac{A_{i}(u)}{z-u_{i}} \mathrm{~d} z
$$

2) $\mathrm{d} \omega=\omega \wedge \omega$.

Proof. Condition 2) is the Frobenius integrability condition for the Pfaffian system $\mathrm{d} Y=\omega Y$. Condition 1) assures that the $z$ part of the Pfaffian system is the Fuchsian system (A.1). If the deformation is isomonodromic, then by Proposition A.2 there is a holomorphic family $Y(z, u)$ and as in the case of (2.5)-(2.6), we see that $\omega=\mathrm{d} Y Y^{-1}$ is single valued and holomorphic on $\mathcal{E}$. This also implies that the Pfaffian system is integrable, then 2) holds. Conversely, if 2) holds, then by the general properties of linear Pfaffian systems there exist a solution $Y$, holomorphic on the universal covering of $\mathcal{E}$, whose monodromy - as in (2.5)-(2.6) - is independent of $u$.

We are going to show below that for a non-resonant Fuchsian system (i.e., the eigenvalues of the matrices $A_{i}$ do not differ by non-zero integers) all weak isomonodromic deformations are actually strong, namely not only the monodromy matrices are constant, but also certain essential monodromy data do not depend on $u$ (see Definition A.9 below). On the other hand, if the system is resonant, there exist both weak and strong isomonodromic deformations, which are inequivalent. To say it in other well known words, in the non-resonant case only Schlesinger deformations exist, while in the resonant case also non-Schlesinger deformations appear. The former are strong (preserving essential data), the latter are weak (preserving only monodromy matrices). First, let us recall Schlesinger deformations.

Schlesinger deformations. For a Fuchsian system

$$
\frac{\mathrm{d} Y}{\mathrm{~d} z}=\sum_{i=1}^{N} \frac{A_{i}^{0}}{z-u_{i}^{0}} Y, \quad \sum_{i=1}^{N} A_{i}^{0}=0,
$$

it is always possible to consider its Schlesinger deformations, given by $\omega=\omega_{s}$, where

$$
\omega_{s}:=\sum_{i=1}^{N} \frac{A_{i}(u)}{z-u_{i}} \mathrm{~d}\left(z-u_{i}\right), \quad A_{i}\left(u^{0}\right)=A_{i}^{0} .
$$

Such deformations exist if and only if there exist $A_{1}(u), \ldots, A_{N}(u)$ such that

$$
\mathrm{d} \omega_{s}=\omega_{s} \wedge \omega_{s}, \quad A_{i}\left(u^{0}\right)=A_{i}^{0}, \quad \sum_{i=1}^{N} A_{i}(u)=0 .
$$


A standard computation shows that the condition $\mathrm{d} \omega_{s}=\omega_{s} \wedge \omega_{s}$ is equivalent to the Schlesinger equations

$$
\mathrm{d} A_{i}(u)=\sum_{j \neq i}\left[A_{j}(u), A_{i}(u)\right] \frac{\mathrm{d}\left(u_{j}-u_{i}\right)}{u_{j}-u_{i}} .
$$

This implies that

$$
\sum_{j=1}^{N} \frac{\partial A_{i}}{\partial u_{j}}=0
$$

which ensures that $\sum_{i} A_{i}(u)=0$ whenever $\sum_{i} A_{i}\left(u^{0}\right)=0$. The Schlesinger system is well known to be Frobenius integrable. We conclude that $\omega_{s}$, called Schlesinger deformation of the Fuchsian system (A.2), always exists, including the resonant case (but it is not the unique one in this case). For an isomonodromic holomorphic fundamental matrix $Y_{s}(z, u)$ of $\omega_{s}$, we have

$$
\mathrm{d}_{u} Y_{s}(\infty, u) Y_{s}(\infty, u)^{-1}=-\left.\sum_{j=1}^{\infty} \frac{A_{i}(u)}{z-u_{i}}\right|_{z=\infty} \mathrm{d} u_{i}=0,
$$

so that $Y_{s}(\infty, u)$ is a constant independent of $u$ (for example $Y_{s}(\infty, u)=I$ ). For this reason, we say that the Schlesinger deformation $\omega_{s}$ is normalized.

Non-normalized Schlesinger deformations [26]. Together with $\omega_{s}$, there always exist deformations

$$
\omega=\omega_{s}+\sum_{i=1}^{N} \gamma_{i}(u) \mathrm{d} u_{i}
$$

with holomorphic matrix coefficients $\gamma_{i}(u)$ on $\mathbb{D}\left(u^{0}\right)$, which are called non-normalized. To see this, let $Y_{s}(z, u)$ be such that $Y_{s}(\infty, z)=I$ (or another constant matrix) and $\mathrm{d} Y_{s}=\omega_{s} Y_{s}$. Then let

$$
\widehat{Y}(z, u):=\Gamma(u) Y_{s}(z, u),
$$

for a holomorphically invertible matrix $\Gamma(u)$ on $\mathbb{D}\left(u^{0}\right)$ (so that $\widehat{Y}(\infty, u)=\Gamma(u)$ and the normalization at $\infty$ is lost). We have

$$
\begin{aligned}
\mathrm{d} \widehat{Y} & =\Gamma \mathrm{d} Y_{s}+\mathrm{d} \Gamma Y_{s}=\Gamma \omega_{s} Y_{s}+\mathrm{d} \Gamma Y_{s} \\
& =\left(\sum_{i=1}^{N} \frac{\Gamma A_{i} \Gamma^{-1}}{z-u_{i}} \mathrm{~d}\left(z-u_{i}\right)+\sum_{i=1}^{N} \frac{\partial \Gamma}{\partial u_{i}} \Gamma^{-1} \mathrm{~d} u_{i}\right) \widehat{Y}=:\left(\widehat{\omega}_{s}+\sum_{i=1}^{N} \gamma_{i}(u) \mathrm{d} u_{i}\right) \widehat{Y} .
\end{aligned}
$$

Conversely, for any $\gamma_{1}, \ldots, \gamma_{N}$ we need to prove that there exists an holomorphically invertible $\Gamma$ such that $\mathrm{d} \Gamma \Gamma^{-1}=\sum_{i} \gamma_{i} \mathrm{~d} u_{i}$, namely

$$
\frac{\partial \Gamma}{\partial u_{i}}=\gamma_{i}(u) \Gamma, \quad i=1, \ldots, N
$$

The integrability condition of this system is

$$
\partial_{j} \gamma_{i}+\gamma_{i} \gamma_{j}=\partial_{i} \gamma_{j}+\gamma_{j} \gamma_{i}
$$

On the other hand, by assumption, $\omega=\omega_{s}+\sum_{i} \gamma_{i} \mathrm{~d} u_{i}$ satisfies $\mathrm{d} \omega=\omega \wedge \omega$. We have

$$
\mathrm{d} \omega=\mathrm{d} \omega_{s}+\sum_{i j} \partial_{j} \gamma_{i} \mathrm{~d} u_{j} \wedge \mathrm{d} u_{i}=\mathrm{d} \omega_{s}+\sum_{i<j}\left(\partial_{i} \gamma_{j}-\partial_{j} \gamma_{i}\right) \mathrm{d} u_{i} \wedge \mathrm{d} u_{j}
$$


and

$$
\omega \wedge \omega=\omega_{s} \wedge \omega_{s}+\sum_{i<j}\left(\gamma_{i} \gamma_{j}-\gamma_{j} \gamma_{i}\right) \mathrm{d} u_{i} \wedge \mathrm{d} u_{j}
$$

Using that $\mathrm{d} \omega_{s}=\omega_{s} \wedge \omega_{s}$, we see that $\mathrm{d} \omega=\omega \wedge \omega$ implies (A.4).

The same considerations as above show that if $\omega$ is a 1-form for an isomonodromic family of Fuchsian systems, then any $\omega+\sum_{i} \gamma_{i}(u) \mathrm{d} u_{i}$ is again responsible for an isomonodromic deformation.

Schlesinger deformations have the following property.

Proposition A.4. Consider the Pfaffian system

$$
\mathrm{d} Y=\left(\sum_{i=1}^{N} \frac{A_{i}(u)}{z-u_{i}} \mathrm{~d}\left(z-u_{i}\right)+\sum_{i=1}^{n} \gamma_{i}(u) \mathrm{d} u_{i}\right) Y,
$$

where the matrices $A_{i}(u)$ and $\gamma_{i}(u)$ are holomorphic in $\mathbb{D}\left(u^{0}\right)$. Then each $A_{i}(u)$ is holomorphically similar to a constant Jordan form $J_{i}$. Namely, there exists $G_{i}(u)$ holomorphic in $\mathbb{D}\left(u^{0}\right)$ such that $J_{i}=G_{i}(u)^{-1} A_{i}(u) G_{i}(u)$ is Jordan and independent of $u$. In particular, the spectrum of each $A_{i}(u)$ is independent of $u$, so that a Schlesinger isomonodromic deformation is isospectral.

Proof. Suppose we want to prove that $J_{1}=G_{1}(u)^{-1} A_{1}(u) G_{1}(u)$ is independent of $u$. As far as the local behaviour around $z-u_{1}=0$ is concerned, (A.5) can be rewritten as

$$
\mathrm{d} Y=\left(\frac{P_{0}(x, u)}{x} \mathrm{~d} x+\sum_{i=1}^{N} P_{i}(x, u) \mathrm{d} u_{i}\right) Y,
$$

where the $N+1$ independent variables are $x:=z-u_{1} ; u_{1}, u_{2}, \ldots, u_{N}$. Each $P_{k}(x, u)$, for $k=0,1, \ldots, N$, is holomorphic in a neighbourhood of $x=z-u_{1}=0$. Explicitly

$$
\begin{aligned}
& \frac{P_{0}}{x}=\frac{A_{1}(u)}{x}+\sum_{j=2}^{N} \frac{A_{j}(u)}{x-\left(u_{j}-u_{1}\right)}, \\
& \sum_{i=1}^{N} P_{i} \mathrm{~d} u_{i}=\sum_{j=2}^{N} \frac{A_{j}(u)}{x-\left(u_{j}-u_{1}\right)}\left(\mathrm{d} u_{1}-\mathrm{d} u_{j}\right)+\sum_{j=1}^{N} \gamma_{j}(u) \mathrm{d} u_{j} .
\end{aligned}
$$

So, the proof repeats the arguments of the proofs of Lemma 2.2 and Proposition 2.3.

We now go back to the most general $\omega$ in Theorem A.3, which we are going to characterise. Let $Y(z, u)$ be a holomorphic weak isomonodromic fundamental matrix solution of $\mathrm{d} Y=\omega Y$. Being in particular a solution of the Fuchsian system $\partial_{z} Y=\sum_{i} A_{i}(u) /\left(z-u_{i}\right) Y$, we write its Levelt form at $z=u_{i}$

$$
Y(z, u)=\widehat{Y}_{i}(z, u)\left(z-u_{i}\right)^{D_{i}}\left(z-u_{i}\right)^{L_{i}(u)} C_{i}(u),
$$

where $C_{i}(u)$ is an invertible connection matrix, and

- $\widehat{Y}_{i}(z, u)$ is holomorphically invertible at $z=u_{i}$, and $\widehat{Y}_{i}\left(z=u_{i}, u\right)=G_{i}(u)$ of Proposition A.4.

- $L_{i}(u)$ is block-diagonal $L_{i}=L_{1}^{(i)} \oplus \cdots \oplus L_{\ell_{i}}^{(i)}$, with upper triangular matrices $L_{q}^{(i)}$; each $L_{q}^{(i)}$ has only one eigenvalue $\sigma_{q}^{(i)}$ satisfying $0 \leq \operatorname{Re} \sigma_{q}^{(i)}<1$, and $\sigma_{p}^{(i)} \neq \sigma_{q}^{(i)}$ for $1 \leq p \neq q \leq \ell_{i}$. 
- $D_{i}$ is a diagonal matrix of integers, which can be split into blocks $D_{1}^{(i)} \oplus \cdots \oplus D_{\ell_{i}}^{(i)}$. The integers $d_{q, r}^{(i)}$ in each $D_{q}^{(i)}=\operatorname{diag}\left(d_{q, 1}^{(i)}, d_{q, 2}^{(i)}, \ldots\right)$ form a non-increasing (finite) sequence $d_{q, 1}^{(i)} \geq d_{q, 2}^{(i)} \geq \cdots$.

- The eigenvalues of $A_{i}$ are $\lambda_{q, s}^{(i)}:=\sigma_{q}^{(i)}+d_{q, s}^{(i)}$. Each block $L_{q}^{(i)}(u)$ corresponds to the eigenvalues of $A_{i}(u)$ which differ by non-zero integers.

- The following holds

$$
\lim _{z \rightarrow u_{i}}\left[\widehat{Y}_{i}(z, u)\left(D_{i}+\left(z-u_{i}\right)^{D_{i}} L_{i}(u)\left(z-u_{i}\right)^{-D_{i}}\right) \widehat{Y}_{i}^{-1}(z, u)\right]=A_{i}(u) .
$$

Lemma A.5 ([9, Lemma 2$])$. $L_{i}(u)$ and $C_{i}(u)$ can be taken holomorphic on $\mathbb{D}\left(u^{0}\right)$, so that also each $\widehat{Y}_{i}(z, u)$ depends holomorphically on $u$.

The following lemma shows that a general deformation of Theorem A.3 is isospectral.

Lemma A.6. For a weak isomonodromic deformation of a Fuchsian system, the diagonal of $L_{i}(u)$ is constant and $D_{i}$ is constant, so that the eigenvalues of each $A_{i}(u)$ do not depend on $u$.

Proof. We rewrite (A.8) as

$$
\begin{aligned}
& Y(z, u)=\widehat{Y}_{i}(z, u)\left(z-u_{i}\right)^{D_{i}} C_{i}(u)\left(z-u_{i}\right)^{\mathcal{L}_{i}}, \\
& \mathcal{L}_{i}:=C_{i}(u)^{-1} L_{i}(u) C_{i}(u) \equiv \frac{1}{2 \pi i} \log M_{i}, \quad 0 \leq \operatorname{Re}\left(\text { Eigenvls of } \mathcal{L}_{i}\right)<1 .
\end{aligned}
$$

Since $\mathrm{d} M_{i}=0$, then $\mathrm{d} \mathcal{L}_{i}=0$. Hence, the eigenvalues $\sigma_{q}^{(i)}$ of $L_{i}(u)$ are constant. Now, the eigenvalues $\lambda_{q, s}^{(i)}(u)=\sigma_{q}^{(i)}+d_{q, s}^{(i)}$ of $A_{i}(u)$ are continuous, being $A_{i}(u)$ continuous, so the integers $d_{q, s}^{(i)}$ are constant.

We observe that if $C_{i}$ and $L_{i}$ are constant, then $\omega=\omega_{s}+\sum_{i} \gamma_{i} \mathrm{~d} u^{i}$, as in (A.3). Indeed, in this case we have

$$
\begin{aligned}
\mathrm{d} Y Y^{-1} & =\mathrm{d} \widehat{Y}_{i} \widehat{Y}_{i}^{-1}+\widehat{Y}_{i} \frac{D_{i}+\left(z-u_{i}\right)^{D_{i}} L_{i}\left(z-u_{i}\right)^{-D_{i}}}{z-u_{i}} \widehat{Y}_{i}^{-1} \mathrm{~d}\left(z-u_{i}\right) \\
& =\widehat{Y}_{i}\left(u_{i}\right) \frac{D_{i}+\lim _{z \rightarrow u_{i}}\left(z-u_{i}\right)^{D_{i}} L_{i}\left(z-u_{i}\right)^{-D_{i}}}{z-u_{i}} \widehat{Y}_{i}^{-1}\left(u_{i}\right) \mathrm{d}\left(z-u_{i}\right)+\text { holomorphic } \\
& =\frac{A_{i}(u)}{z-u_{i}} \mathrm{~d}\left(z-u_{i}\right)+\text { holomorphic. }
\end{aligned}
$$

The holomorphic part is regular at $z=u_{i}$, and moreover $\mathrm{d} Y Y^{-1}=\mathrm{d} \Gamma \Gamma^{-1}$ at $z=\infty$, having fixed $\Gamma(u):=Y(\infty, u)$. Thus, Liouville theorem yields $\omega=\omega_{s}+\mathrm{d} \Gamma \Gamma^{-1}$, which is (A.3). The converse of the above is also true:

Lemma A.7 ([7, 9, 28, 34]). If the deformation is Schlesinger, i.e., $\omega=\omega_{s}$, or its nonnormalized version (A.3), then $C_{i}$ and $L_{i}$ are constant.

Proof. We prove that $L_{1}$ and $C_{1}$ are constant, being the other cases analogous. The Pfaffian system is rewritten in the form (A.6)-(A.7). Then, by [34, Theorem 5], there exists a gauge transformation

$$
Y=V(x, u) \tilde{Y},
$$


holomorphic in a neighbourhood of $(x, u)=\left(0, u^{0}\right)$, given by a convergent series

$$
V(x, u)=I+\sum_{k_{1}+\cdots+k_{N}>0} V_{k} x^{k_{0}}\left(u_{1}-u_{1}^{0}\right)^{k_{1}} \cdots\left(u_{N}-u_{N}^{0}\right)^{k_{N}},
$$

with $k=\left(k_{0}, k_{1}, \ldots, k_{N}\right)$, such that the Pfaffian system is transformed to

$$
\mathrm{d} \widetilde{Y}=\left(\frac{P_{0}\left(0, u^{0}\right)}{x} \mathrm{~d} x\right) \widetilde{Y} \equiv\left[\left(\frac{A_{1}\left(u^{0}\right)}{x}+\sum_{j=2}^{N} \frac{A_{j}\left(u^{0}\right)}{x-\left(u_{j}^{0}-u_{1}^{0}\right)}\right) \mathrm{d} x\right] \tilde{Y} .
$$

Thus, $\widetilde{Y}$ satisfies an $n \times n$ Fuchsian system in variable $x$, independent of parameters (being $u^{0}$ fixed), so that there is a fundamental matrix solutions in Levelt form at $x=0$, independent of $u$,

$$
\widetilde{Y}_{1}(x):=W(x) x^{D_{1}} x^{L_{1}}, \quad W(x)=W_{0}+\sum_{\ell=1}^{\infty} W_{\ell} x^{\ell},
$$

the series being convergent and holomorphic at $x=0$. Hence, there is a fundamental matrix solution of the initial system of the form

$$
Y_{1}(z, u)=V(x, u) W(x) x^{D_{1}} x^{L_{1}} \equiv \widehat{Y}_{1}(z, u)\left(z-u_{1}\right)^{D_{1}}\left(z-u_{1}\right)^{L_{1}},
$$

where

$$
\begin{aligned}
& \widehat{Y}_{1}(z, u):=\left.V(x, u) W(x)\right|_{x=z-u_{1}} \\
& =\left(I+\sum_{k_{1}+\cdots+k_{N}>0} V_{k}\left(z-u_{1}\right)^{k_{0}}\left(u_{1}-u_{1}^{0}\right)^{k_{1}} \cdots\left(u_{N}-u_{N}^{0}\right)^{k_{N}}\right)\left(W_{0}+\sum_{\ell=1}^{\infty} W_{\ell}\left(z-u_{1}\right)^{\ell}\right) .
\end{aligned}
$$

The latter can be rewritten as a convergent saries at $z=u_{1}$ with coefficients holomorphic in $u$ :

$$
\widehat{Y}_{1}(z, u)=G_{1}(u)\left(I+\sum_{\ell=1}^{\infty} \Psi_{\ell}(u)\left(z-u_{1}\right)^{\ell}\right), \quad G_{1}(u)=V(0, u) W_{0} .
$$

Notice that $G_{1}(u)$ puts $A_{1}(u)$ in Jordan form as in Proposition A.4. The proof is concluded observing that $Y_{1}(z, u)$ solves the initial Pfaffian system, so that also $Y(z, u)=Y_{1}(z, u) C_{1}$ is a fundamental solution if and only if $C_{1}$ is constant. The proof can also be deduced directly from [34, Theorems 4 and 7].

From the above, we have the following

Proposition A.8. The deformation in Theorem A.3 is Schlesinger, namely $\omega=\omega_{s}$, or its non-normalized version (A.3), if and only if the Pfaffian system admits an isomonodromic fundamental matrix (A.8) such that $L_{i}$ and $C_{i}$ are constant.

Definition A.9. The essential monodromy data associated with a weakly isomonodromic $Y(z, u)$ are the matrices

$$
D_{j}, L_{j}, C_{j}, \quad j=1, \ldots, N .
$$

Definition A.10. We call $Y(z, u)$ as strongly isomonodromic fundamental matrix if the essential monodromy data are constant on $\mathbb{D}\left(u^{0}\right)$. Equivalently, each $Y_{j}(z, u):=\widehat{Y}_{j}(z, u)\left(z-u_{j}\right)^{D_{j}}(z-$ $\left.u_{j}\right)^{L_{j}}$ is a fundamental matrix of $\mathrm{d} Y=\omega Y$. 
Remark A.11. Recall that the $D_{j}$ are always constant for a weak isomonodromic deformation (Lemma A.6). Moreover,

$$
\mathrm{d} C_{j}=0 \quad \text { implies } \quad \mathrm{d} L_{j}=0,
$$

because we can write the monodromy matrices as $M_{j}=C_{j}^{-1} e^{2 \pi i L_{j}} C_{j}$. Thus, one can define a strong deformation to be one such that all the matrices $C_{j}$ are constant, in the same way as in Definition 3.1 (the connection matrices $C_{j}$ play the same role of the matrices $H_{r}$ ).

Thus, we conclude that

Proposition A.12 (Proposition A.8 revised). An isomonodromic deformation is strong if and only if it is a Schlesinger deformation or its non-normalized version (A.3).

Following [28], we may call $\mathcal{P}_{i}(z, u):=\left(z-u_{i}\right)^{D_{i}}\left(z-u_{i}\right)^{L_{i}(u)} C_{i}(u)$ the principal part of $Y(z, u)$ at $z=u_{i}$, so that

$$
Y(z, u)=\widehat{Y}_{i}(z, u) \mathcal{P}_{i}(z, u) .
$$

In case $C_{i}$ and $L_{i}$ are constant, then

$$
\mathcal{P}_{i}(z, u) \equiv \mathcal{P}_{i}\left(z-u_{i}\right)
$$

namely, $\mathcal{P}_{i}$ depends on $(z, u)$ only through the combination $\left(z-u_{i}\right)$. If such a dependence occurs at any $u_{i}$, the deformation is called isoprincipal in [28]. The main result of [28] is that a family of Fuchsian systems (A.1) is an isoprincipal deformation if and only if the matrices $A_{i}$ satisfy the Schlesinger equations. This result is equivalent to Proposition A.8.

We are ready to review the most general form of $\omega$.

Theorem A.13 (non-Schesinger deformations, Bolibruch $[8,9]$ ). All the possible matrix differential 1-form of Theorem A.3, holomorphic on $\mathbb{P}^{1} \times \mathbb{D}\left(u^{0}\right) \backslash \bigcup_{i=1}^{n}\left\{z-u_{i}=0\right\}$, which give an isomonodromic family of Fuchsian systems (A.1), have the form

$$
\omega=\omega_{s}+\sum_{i=1}^{N} \gamma_{i}(u) \mathrm{d} u_{i}+\sum_{i=1}^{N} \sum_{j=1}^{N} \sum_{k=1}^{m_{i}} \frac{\gamma_{i j k}(u)}{\left(z-u_{i}\right)^{k}} \mathrm{~d} u_{j} .
$$

where $\gamma_{i}(u), \gamma_{i j k}(u)$ are holomorphic on $\mathbb{D}\left(u^{0}\right)$ and $m_{i}$ is the maximal integer difference of eigenvalues of $A_{i}(u)$.

Proof. The proof is in [8] and [9]. We take the Schlesinger deformation $\omega_{s}$ with the same initial condition $A_{i}^{0}=A_{i}\left(u^{0}\right)$ of $\omega$, namely

$$
\left.\omega_{s}\right|_{u^{0}}=\left.\omega\right|_{u^{0}} .
$$

Let $Y_{s}(z, u)$ be an isomonodromic fundamental matrix for $\omega_{s}$. By isomonodromicity, $Y(z, u)$ and $Y_{s}(z, u)$ have the same monodromy matrices, which are those of

$$
\frac{\mathrm{d} Y}{\mathrm{~d} z}=\sum_{i=1}^{N} \frac{A_{i}^{0}}{z-u_{i}^{0}} Y .
$$

Hence, $\Gamma(z, u):=Y(z, u) Y_{s}(z, u)^{-1}$ is single valued and meromorphic on $\mathbb{P}^{1} \times \mathbb{D}\left(u^{0}\right)$, with poles at $z=u_{i}, 1 \leq i \leq N$. Moreover $\omega=\mathrm{d} \Gamma \Gamma^{-1}+\Gamma \omega_{s} \Gamma^{-1}$. This expression does show that $\omega$ has the structure (A.9). In order to predict $m_{i}$, we write

$$
Y(z, u)=\widehat{Y}_{i}(z, u)\left(z-u_{i}\right)^{D_{i}}\left(z-u_{i}\right)^{L_{i}(u)} C_{i}(u),
$$




$$
Y_{s}(z, u)=\widehat{Y}_{i}^{s}(z, u)\left(z-u_{i}\right)^{D_{i}}\left(z-u_{i}\right)^{L_{i}^{s}} C_{i}^{s}
$$

where $L_{i}^{s}$ and $C_{i}^{s}$ are constant, by Proposition A.8. Being the monodromy $M_{i}$ of $Y$ and $Y_{s}$ the same, we have

$$
C_{i}(u)^{-1} L_{i}(u) C_{i}(u)=C_{i}^{s-1} L_{i}^{s} C_{i}^{s} .
$$

Thus,

$$
L_{i}(u)=\mathcal{R}_{i} L_{i}^{s} \mathcal{R}_{i}^{-1}, \quad \mathcal{R}_{i}:=C_{i}(u) C_{i}^{s-1},
$$

and $\mathcal{R}_{i}$ must have the same block structure of $L_{i}(u)$ and $L_{i}^{s}$. Moreover,

$$
Y(z, u)=\widehat{Y}_{i}(z, u)\left(z-u_{i}\right)^{D_{i}} \mathcal{R}_{i}\left(z-u_{i}\right)^{L_{i}^{s}} C_{i}^{s} .
$$

Thus, we have

$$
\begin{aligned}
\mathrm{d} Y Y^{-1}= & \mathrm{d} \widehat{Y}_{i} \widehat{Y}_{i}^{-1}+\widehat{Y}_{i}\left(\frac{D_{i}+\left(z-u_{i}\right)^{D_{i}} \mathcal{R}_{i} E_{i}^{s} \mathcal{R}_{i}^{-1}\left(z-u_{i}\right)^{-D_{i}}}{z-u_{i}}\right) \widehat{Y}_{i}^{-1} \mathrm{~d}\left(z-u_{i}\right) \\
& +\widehat{Y}_{i}\left(z-u_{i}\right)^{D_{i}} \mathrm{~d} \mathcal{R}_{i} \mathcal{R}_{i}^{-1}\left(z-u_{i}\right)^{-D_{i}} \widehat{Y}_{i}^{-1} \\
= & \mathrm{d} \widehat{Y}_{i} \widehat{Y}_{i}^{-1}+\widehat{Y}_{i}\left(\frac{D_{i}+\left(z-u_{i}\right)^{D_{i}} L_{i}(u)\left(z-u_{i}\right)^{-D_{i}}}{z-u_{i}}\right) \widehat{Y}_{i}^{-1} \mathrm{~d}\left(z-u_{i}\right) \\
& +\widehat{Y}_{i}\left(z-u_{i}\right)^{D_{i}} \mathrm{~d} \mathcal{R}_{i} \mathcal{R}_{i}^{-1}\left(z-u_{i}\right)^{-D_{i}} \widehat{Y}_{i}^{-1} \\
= & \text { holomorphic }+\frac{A_{i}(u)}{z-u_{i}} \mathrm{~d}\left(z-u_{i}\right)+\widehat{Y}_{i}\left(z-u_{i}\right)^{D_{i}} \mathrm{~d} \mathcal{R}_{i} \mathcal{R}_{i}^{-1}\left(z-u_{i}\right)^{-D_{i}} \widehat{Y}_{i}^{-1} .
\end{aligned}
$$

The last term $\widehat{Y}_{i}\left(z-u_{i}\right)^{D_{i}} \mathrm{~d} \mathcal{R}_{i} \mathcal{R}_{i}^{-1}\left(z-u_{i}\right)^{-D_{i}} \widehat{Y}_{i}^{-1}$ contains matrix entries with poles at $\left(z-u_{i}\right)=0$, plus holomorphic terms. This proves again, by Liouville theorem, that $\omega$ has the structure (A.9). The block diagonal structure of $\mathcal{R}_{i}$, being the same as that of $D_{i}=D_{1}^{(i)} \oplus \cdots \oplus D_{\ell_{i}}^{(i)}$, assures that $m_{i}$ is the maximum over $q$ of the maximal difference of the eigenvalues of $D_{q}^{(i)}, q=1,2, \ldots, \ell_{i}$. This is precisely the maximal integer difference of eigenvalues of $A_{i}(u)$.

In the non-resonant case, $m_{i}=0$ for every $i=1, \ldots, N$. Therefore, we obtain the following

Corollary A.14. If for every $i=1, \ldots, N$ the matrices $A_{i}$ are non-resonant, then every $\omega$ of Theorem A.3, defining an isomonodromic family of Fuchsian systems (A.1), is of the form

$$
\omega=\omega_{s}+\sum_{i=1}^{n} \gamma_{i}(u) \mathrm{d} u_{i} .
$$

In other words, the isomonodromy deformation of a non-resonant Fuchsian system are only the Schlessinger deformations, possibly non-normalized. The term $\sum_{i=1}^{N} \sum_{j=1}^{N} \sum_{k=1}^{m_{i}} \frac{\gamma_{i j k}(u)}{\left(z-u_{i}\right)^{k}} \mathrm{~d} u_{j}$ can only appear in the resonant case. Summarizing, we have found that

1) in the non-resonant case, the definitions of weak and strong isomonodromic deformation are equivalent, and the Schlesinger equations are necessary and sufficient conditions;

2 ) in the resonant case, there exist both weak isomonodromic deformations, determined by the form $\omega$ in Theorem A.13 and called non-Schlesinger, and strong isomonodromic deformations, which are Schlesinger deformations determined by $\omega_{s}$ (or its non-normalized version). 
There are examples of non-Schlesinger deformations in $[6,9,29] .{ }^{9}$ A simple example is given at the end of [28], and we report it here. Consider the family of differential systems

$$
\begin{aligned}
\frac{\mathrm{d} Y}{\mathrm{~d} z}= & \left(\frac{1}{z-u}\left(\begin{array}{ll}
1 & 0 \\
0 & 0
\end{array}\right)+\frac{1}{z-1}\left(\begin{array}{cc}
-1 & \frac{-u(u-1) h(u)}{u-3} \\
0 & 0
\end{array}\right)+\frac{1}{z-2}\left(\begin{array}{cc}
0 & \frac{2 u(u-2) h(u)}{u-3} \\
0 & 1
\end{array}\right)\right. \\
& \left.+\frac{1}{z-3}\left(\begin{array}{cc}
0 & -u h(u) \\
0 & -1
\end{array}\right)\right) Y .
\end{aligned}
$$

A fundamental matrix solution is

$$
Y(z, u)=\left(\begin{array}{cc}
\frac{z-u}{z-1} & \frac{-2 t(z-u) h(u)}{(z-1)(z-3)(u-3)} \\
0 & \frac{z-2}{z-3}
\end{array}\right)
$$

Here $h(u)$ is an arbitrary function, which is holomorphic at $u=0$. The system is resonant at all the Fuchsian singularities $z=u, 1,2,3$. One can check that the residue matrices do not satisfy the Schlesinger equations, but the $u$-deformation is isomonodromic in the weak sense, because $Y(z, u)$ is single-valued and hence has trivial monodromy matrices $M_{i}=I:=\operatorname{diag}(1,1)$. For $z$ close to $u$, we have

$$
Y(z, u)=\left(\begin{array}{cc}
\frac{1}{u-1} & 0 \\
0 & \frac{u-2}{u-3}
\end{array}\right)(I+O(z-u))\left(\begin{array}{cc}
z-u & 0 \\
0 & 1
\end{array}\right)
$$

with constant connection matrix $=I$. This is not the case at other poles of the system. For example, for $z$ close to 1 , we have

$$
Y(z, u)=\left(\begin{array}{cc}
\frac{u(1-u) h(u)}{u-3} & 1 \\
1 & 0
\end{array}\right)(I+O(z-1))\left(\begin{array}{cc}
1 & 0 \\
0 & (z-1)^{-1}
\end{array}\right)\left(\begin{array}{cc}
0 & 1 / 2 \\
1-u & \frac{u(1-u) h(u)}{u-3}
\end{array}\right),
$$

with $u$-dependent connection matrix

$$
C_{1}(u)=\left(\begin{array}{cc}
0 & 1 / 2 \\
1-u & \frac{u(1-u) h(u)}{u-3}
\end{array}\right) .
$$

So, the deformation is not isomonodromic in the strong - or isoprincipal - sense. The monodromy matrix is nevertheless constant and equal to $I$.

As noted in [28], the example shows that a weak isomonodromic deformation may not have the Painlevé property. Indeed, in the above example we can choose $h(u)$ arbitrarily (provided it is holomorphic at $u=0$ ). On the other hand, if the deformation is isomonodromic in the strong sense, namely Schlesinger, then the Painlevé property holds also in the resonant case.

\section{B Proof of Proposition 2.6}

We recall that a small polydisc $\mathbb{D}^{\prime}$ has been introduced by Sibuya to guarantee that the fundamental matrices $Y_{r}(z, u)$ in $(2.17)$ are holomorphic of $u$.

\footnotetext{
${ }^{9}$ One can also have a look at [30], though the question there is concerned with Schlesinger transformations that also shift the eigenvalues.
} 
Lemma B.1. Let $\omega_{0}(x)=\left(\Lambda+\frac{A}{z}\right)$ be as in $(2.3)$, let $\omega_{1}, \ldots, \omega_{n}$ be holomorphic of $(z, u) \in$ $\mathbb{C} \times \mathbb{D}\left(u^{0}\right)$, and $A(u)$ holomorphic on $\mathbb{D}\left(u^{0}\right)$. Assume that $z=\infty$ is at most a pole of $\omega_{1}, \ldots, \omega_{n}$. Then, for $u \in \mathbb{D}^{\prime}$, the Pfaffian system has the following structure

$$
\omega=\left(\Lambda+\frac{A}{z}\right) \mathrm{d} z+\sum_{j=1}^{n}\left(z E_{j}+\omega_{j}(0, u)\right) \mathrm{d} u_{j},
$$

with

$$
\omega_{j}(0, u)=\left[F_{1}(u), E_{j}\right]+\mathcal{D}_{j}(u),
$$

where $F_{1}$, appearing in the formal expansion (2.16), is given explicitly in (2.21), and

$$
\mathcal{D}_{j}(u)=\frac{\partial H_{r}(u)}{\partial u_{j}} H_{r}(u)^{-1}
$$

is a diagonal matrix independent of $r \in \mathbb{Z}$ and holomorphic on $u \in \mathbb{D}^{\prime}$. Moreover,

$$
\mathrm{d} B=0 .
$$

Proof. We write

$$
\omega=\mathrm{d} Y Y^{-1} \text {. }
$$

where $Y(z, u)=Y^{(0)}(z, u) H$ is a holomorphic isomonodromic family. Recalling that $D$ is constant and $\mathrm{d} L=\mathrm{d} H=0$ (one can take directly $H=I$ ), we have

$$
\omega_{j}=\frac{\partial Y}{\partial u_{j}} Y^{-1}=\frac{\partial \widehat{Y}^{(0)}}{\partial u_{j}}\left(\widehat{Y}^{(0)}\right)^{-1}=\operatorname{holom}(z, u),
$$

where $\operatorname{holom}(z, u)$ is some convergent Taylor series at $z=0$, of order $\mathcal{O}(1)$ for $z \rightarrow 0$, with holomorphic coefficients depending on $u \in \mathbb{D}\left(u^{0}\right)$. Keeping into account the explicit form (2.12) of $\widehat{Y}^{(0)}$ (in particular, recall that $G(u)$ satisfies (2.8) as in Proposition 2.3), we have

$$
\omega_{j}=\partial_{j} G G^{-1}+\mathcal{O}(z) \equiv \omega_{j}(0, u)+\mathcal{O}(z) .
$$

Successively, we compute the representation of $\omega_{j}$ close to $z=\infty$, using (2.19),

$$
\begin{aligned}
\omega_{j} & =\frac{\partial Y}{\partial u_{j}} Y^{-1} \\
& =\frac{\partial \widehat{Y}_{r}}{\partial u_{j}} \widehat{Y}_{r}^{-1}+\widehat{Y}_{r} \frac{\partial B}{\partial u_{j}} \widehat{Y}_{r}^{-1} \log z+z \widehat{Y}_{r} E_{j} \widehat{Y}_{r}^{-1}+\widehat{Y}_{r} z^{B}\left(e^{z \Lambda} \frac{\partial H_{r}}{\partial u_{j}} H_{r}^{-1} e^{-z \Lambda}\right) z^{-B} \widehat{Y}_{r}^{-1} .
\end{aligned}
$$

We recall that $u_{i} \neq u_{j}$ for $i \neq j$ and observe that $e^{\left(u_{i}-u_{j}\right) z}$ diverges for $z \rightarrow \infty$ in some subsector of $\mathcal{S}_{r}\left(\overline{\mathbb{D}^{\prime}}\right)$, because the central angular opening is more than $\pi$. Hence

$$
e^{z \Lambda} \frac{\partial H_{r}}{\partial u_{j}} H_{r}^{-1} e^{-z \Lambda} \quad \text { does not diverge exponentially } \Longleftrightarrow \frac{\partial H_{r}}{\partial u_{j}} H_{r}^{-1} \quad \text { is diagonal. }
$$

Since $\Lambda$ and $B$ are also diagonal, we have

$$
\omega_{j}=\left(\frac{\partial \widehat{Y}_{r}}{\partial u_{j}} \widehat{Y}_{r}^{-1}+z \widehat{Y}_{r} E_{j} \widehat{Y}_{r}^{-1}+\widehat{Y}_{r} \frac{\partial H_{r}}{\partial u_{j}} H_{r}^{-1} \widehat{Y}_{r}^{-1}\right)+\widehat{Y}_{r} \frac{\partial B}{\partial u_{j}} \widehat{Y}_{r}^{-1} \log z .
$$


We have at most a pole at $z=\infty$ if and only if

$$
\frac{\partial B}{\partial u_{j}}=0
$$

Then, the asymptotic behaviour (2.16) of $\widehat{Y}_{r}$ implies that

$$
\omega_{j}=z \widehat{Y}_{r} E_{j} \widehat{Y}_{r}^{-1}+\widehat{Y}_{r} \frac{\partial H_{r}}{\partial u_{j}} H_{r}^{-1} \widehat{Y}_{r}^{-1}+\frac{\partial \widehat{Y}_{r}}{\partial u_{j}} \widehat{Y}_{r}^{-1}=z E_{j}+\left[F_{1}, E_{j}\right]+\frac{\partial H_{r}}{\partial u_{j}} H_{r}^{-1}+\mathcal{O}\left(\frac{1}{z}\right)
$$

in the sector $\mathcal{S}_{r}\left(\overline{\mathbb{D}^{\prime}}\right)$. The asymptotics holds for every $r \in \mathbb{Z}$. Now, the asymptotic expansion of a function (in our case $\omega_{j}$ ) on a sector is unique. Since $\mathcal{S}_{r}\left(\overline{\mathbb{D}^{\prime}}\right) \cap \mathcal{S}_{r+1}\left(\overline{\mathbb{D}^{\prime}}\right)$ is not empty, this implies that $\omega_{j}$ has the same expansion in every sector $\mathcal{S}_{r}\left(\overline{\mathbb{D}^{\prime}}\right), \forall r$. In particular, $\partial_{j} H_{r} H_{r}^{-1}$ cannot depend on $r$. We set

$$
\mathcal{D}_{j}(u):=\frac{\partial H_{r}(u)}{\partial u_{j}} H_{r}^{-1}(u) \quad \forall r \in \mathbb{Z} .
$$

Moreover, being $\omega_{j}$ single valued, the asymptotic series represented by $\mathcal{O}\left(\frac{1}{z}\right)$ above, must be a convergent Taylor expansion. In conclusion

$$
\omega_{j}-\left(z E_{j}+\left[F_{1}, E_{j}\right]+\mathcal{D}_{j}\right)=\mathcal{O}\left(\frac{1}{z}\right) \quad \text { is holomorphic at } z=\infty .
$$

Keeping into account (B.1) and Liouville theorem, we obtain that

$$
\omega_{j}-\left(z E_{j}+\left[F_{1}, E_{j}\right]+\mathcal{D}_{j}\right)=0,
$$

as we wanted to prove.

Lemma B.2. Consider a Pfaffian system $\mathrm{d} Y=\omega Y$, and assume that $\omega$ has the following structure

$$
\omega=\left(\Lambda+\frac{A(u)}{z}\right) \mathrm{d} z+\sum_{j=1}^{n}\left(z E_{j}+\omega_{j}(0, u)\right) \mathrm{d} u_{j},
$$

where $A(u)$ and $\omega_{1}(0, u), \ldots, \omega_{n}(0, u)$ are holomorphic on $\mathbb{D}\left(u^{0}\right)$. Then, the Frobenius integrability conditions (2.4) are equivalent to 1) and 2) below:

1) $\omega_{j}(0, u)$ satisfies the constraint

$$
\left[\Lambda, \omega_{j}(0, u)\right]=\left[E_{j}, A\right]
$$

2) A(u) satisfies the non-linear system

$$
\frac{\partial A}{\partial u_{j}}=\left[\omega_{j}(0, u), A\right], \quad j=1,2, \ldots, n,
$$

which is Frobenius integrable.

The above (B.2) determines $\omega_{j}(0, u)$ up to a diagonal matrix $\mathcal{D}_{j}(u)$, as follows

$$
\omega_{j}(0, u)=\left(\frac{A_{a b}(u)\left(\delta_{a j}-\delta_{b j}\right)}{u_{a}-u_{b}}\right)_{a, b=1}^{n}+\mathcal{D}_{j}(u) .
$$

Moreover, the diagonal matrix $B:=\operatorname{diag}\left(A_{11}, \ldots, A_{n n}\right)$ is constant and

$$
\mathcal{D}_{j}=\frac{\partial \mathcal{D}}{\partial u_{j}},
$$

where $\mathcal{D}$ is a matrix whose diagonal only depends on $u \in \mathbb{D}\left(u^{0}\right)$. 
Proof. For brevity, let us write $\omega_{j}(0)$ in place of $\omega_{j}(0, u)$. As in the proof of Lemma 2.2, consider (2.4) for $\beta=0, \alpha=j=1, \ldots, n$ :

$$
\frac{\partial}{\partial u_{j}}\left(\Lambda+\frac{A}{z}\right)+\left(\Lambda+\frac{A}{z}\right)\left(z E_{j}+\omega_{j}(0)\right)=\frac{\partial}{\partial z}\left(z E_{j}+\omega_{j}(0)\right)+\left(z E_{j}+\omega_{j}(0)\right)\left(\Lambda+\frac{A}{z}\right) .
$$

Since $\partial_{j} \Lambda=E_{j}$, we have

$$
E / j+\frac{\partial_{j} A}{z}+z \Lambda E_{j}+\Lambda \omega_{j}(0)+A E_{j}+\frac{A \omega_{j}(0)}{z}=E / j+z E_{j} \Lambda+E_{j} A+\omega_{j}(0) \Lambda+\frac{\omega_{j}(0) A}{z} .
$$

The above identity is equivalent to (B.2) and (B.3). Consider now (2.4) for $\alpha=k, \beta=j \in$ $\{1, \ldots, n\}$ :

$$
\begin{aligned}
& \frac{\partial}{\partial u_{k}}\left(z E_{j}+\omega_{j}(0)\right)+\left(z E_{j}+\omega_{j}(0)\right)\left(z E_{k}+\omega_{k}(0)\right) \\
& \quad=\frac{\partial}{\partial u_{j}}\left(z E_{k}+\omega_{k}(0)\right)+\left(z E_{k}+\omega_{k}(0)\right)\left(z E_{j}+\omega_{j}(0)\right),
\end{aligned}
$$

so that

$$
\begin{aligned}
& \frac{\partial \omega_{j}(0)}{\partial u_{k}}+\underline{z^{2} E_{j} E_{k}}+z\left(E_{j} \omega_{k}(0)+\omega_{j}(0) E_{k}\right)+\omega_{j}(0) \omega_{k}(0) \\
& =\frac{\partial \omega_{k}(0)}{\partial u_{j}}+z^{2} E_{k} E_{j}+z\left(E_{k} \omega_{j}(0)+\omega_{k}(0) E_{j}\right)+\omega_{k}(0) \omega_{j}(0) .
\end{aligned}
$$

Identifying the coefficients of the same powers of $z$ we get

$$
\begin{aligned}
& {\left[E_{j}, \omega_{k}(0)\right]=\left[E_{k}, \omega_{j}(0)\right],} \\
& \frac{\partial \omega_{j}(0)}{\partial u_{k}}+\omega_{j}(0) \omega_{k}(0)=\frac{\partial \omega_{k}(0)}{\partial u_{j}}+\omega_{k}(0) \omega_{j}(0), \quad 1 \leq j \neq k \leq n .
\end{aligned}
$$

(B.6) is the Frobenius integrability condition of system (B.3), as it is shown in the proof of Lemma 2.2. Explicitly writing (B.2), a simple calculation yields (B.4). Now, (B.5) is identically satisfied. Substitution of (B.4) into (B.6) shows, by considering the diagonal, that

$$
\frac{\partial \mathcal{D}_{j}}{\partial u_{k}}-\frac{\partial \mathcal{D}_{k}}{\partial u_{j}}=0, \quad j \neq k
$$

so that the matrix valued form $\sum_{j=1}^{n} \mathcal{D}_{j}(u) \mathrm{d} u_{j}$ is closed. Being the domain simply connected, it is also exact. Substitution of (B.4) into (B.3) and direct calculation shows that

$$
\frac{\partial A_{k k}}{\partial u_{1}}=\cdots=\frac{\partial A_{k k}}{\partial u_{n}}=0, \quad \forall k=1, \ldots, n .
$$

Proof of Proposition 2.6. The assumption that $\omega_{j}(z, u)$ has at most a pole at $z=\infty$ implies that Lemma B.1 applies for $u \in \mathbb{D}^{\prime}$. Hence

$$
\omega_{j}(z, u)=z E_{j}+\left[F_{1}(u), A(u)\right]+\mathcal{D}_{j}(u) \quad \text { for } u \in \mathbb{D}^{\prime} .
$$

Now, Lemma B.2 applies on $\mathbb{D}^{\prime}$. On the other hand, $\omega_{j}(z, u)$ and $z E_{j}+\left[F_{1}(u), A(u)\right]$ are holomorphic on $\mathbb{D}\left(u^{0}\right)$, therefore (B.7) holds on $\mathbb{D}\left(u^{0}\right)$. All the other statements of Proposition 2.6 follow from Lemmas B.1 and B.2. 


\section{Acknowledgements}

I am very grateful to the referee for valuable suggestions which improved the paper, especially concerning the importance of reference [34] for the characterisation of weak and strong isomonodromic deformations.

\section{References}

[1] Anosov D.V., Bolibruch A.A., The Riemann-Hilbert problem, Aspects of Mathematics, Vol. 22, Friedr. Vieweg \& Sohn, Braunschweig, 1994.

[2] Balser W., Jurkat W.B., Lutz D.A., Birkhoff invariants and Stokes' multipliers for meromorphic linear differential equations, J. Math. Anal. Appl. 71 (1979), 48-94.

[3] Balser W., Jurkat W.B., Lutz D.A., A general theory of invariants for meromorphic differential equations. I. Formal invariants, Funkcial. Ekvac. 22 (1979), 197-221.

[4] Balser W., Jurkat W.B., Lutz D.A., A general theory of invariants for meromorphic differential equations. II. Proper invariants, Funkcial. Ekvac. 22 (1979), 257-283.

[5] Balser W., Jurkat W.B., Lutz D.A., On the reduction of connection problems for differential equations with an irregular singular point to ones with only regular singularities. I, SIAM J. Math. Anal. 12 (1981), 691-721.

[6] Bibilo Yu., Filipuk G., Middle convolution and non-Schlesinger deformations, Proc. Japan Acad. Ser. A Math. Sci. 91 (2015), 66-69.

[7] Bolibruch A.A., The fundamental matrix of a Pfaffian system of Fuchs type, Math. USSR Izv. 11 (1977), 1031-1054.

[8] Bolibruch A.A., On isomonodromic deformations of Fuchsian systems, J. Dynam. Control Systems 3 (1997), 589-604.

[9] Bolibruch A.A., On isomonodromic confluences of Fuchsian singularities, Proc. Steklov Inst. Math. 221 (1998), 117-132.

[10] Cotti G., Coalescence phenomenon of quantum cohomology of Grassmannians and the distribution of prime numbers, arXiv:1608.06868.

[11] Cotti G., Dubrovin B., Guzzetti D., Isomonodromy deformations at an irregular singularity with coalescing eigenvalues, Duke Math. J., to appear, arXiv:1706.04808.

[12] Cotti G., Dubrovin B., Guzzetti D., Local moduli of semisimple frobenius coalescent structures, arXiv:1712.08575.

[13] Cotti G., Dubrovin B., Guzzetti D., Helix structures in quantum cohomology of Fano varieties, in preparation.

[14] Cotti G., Guzzetti D., Analytic geometry of semisimple coalescent Frobenius structures, Random Matrices Theory Appl. 6 (2017), 1740004, 36 pages.

[15] Cotti G., Guzzetti D., Results on the extension of isomonodromy deformations to the case of a resonant irregular singularity, Random Matrices Theory Appl., to appear.

[16] Dubrovin B., Geometry of 2D topological field theories, in Integrable Systems and Quantum Groups (Montecatini Terme, 1993), Lecture Notes in Math., Vol. 1620, Springer, Berlin, 1996, 120-348, hep-th/9407018.

[17] Dubrovin B., Geometry and analytic theory of Frobenius manifolds, Proceedings of the International Congress of Mathematicians, Vol. II (Berlin, 1998), Doc. Math. (1998), Extra Vol. II, 315-326, math.AG/9807034.

[18] Dubrovin B., Painlevé transcendents in two-dimensional topological field theory, in The Painlevé Property, Editor R. Conte, CRM Ser. Math. Phys., Springer, New York, 1999, 287-412, math.AG/9803107.

[19] Dubrovin B., On almost duality for Frobenius manifolds, in Geometry, Topology, and Mathematical Physics, Amer. Math. Soc. Transl. Ser. 2, Vol. 212, Amer. Math. Soc., Providence, RI, 2004, 75-132, math.DG/0307374.

[20] Galkin S., Golyshev V., Iritani H., Gamma classes and quantum cohomology of Fano manifolds: gamma conjectures, Duke Math. J. 165 (2016), 2005-2077, arXiv:1404.6407.

[21] Gantmacher F.R., The theory of matrices, Vol. 1, AMS Chelsea Publishing, Providence, RI, 1998. 
[22] Guzzetti D., Deformations with a resonant irregular singularity, in Proceedings of the Workshop Formal and Analytic Solutions of Differential Equations (Alcalá, September 4-8, 2017), to appear.

[23] Guzzetti D., On Stokes matrices in terms of connection coefficients, Funkcial. Ekvac. 59 (2016), 383-433, arXiv:1407.1206.

[24] Haraoka Y., Linear differential equations in the complex domain, Suugaku Shobou, Tokyo, 2015 (in Japanese).

[25] Hsieh P.-F., Sibuya Y., Note on regular perturbations of linear ordinary differential equations at irregular singular points, Funkcial. Ekvac. 8 (1966), 99-108.

[26] Iwasaki K., Kimura H., Shimomura S., Yoshida M., From Gauss to Painlevé. A modern theory of special functions, Aspects of Mathematics, Vol. 16, Friedr. Vieweg \& Sohn, Braunschweig, 1991.

[27] Jimbo M., Miwa T., Ueno K., Monodromy preserving deformation of linear ordinary differential equations with rational coefficients. I. General theory and $\tau$-function, Phys. D 2 (1981), 306-352.

[28] Katsnelson V., Volok D., Deformations of Fuchsian systems of linear differential equations and the Schlesinger system, Math. Phys. Anal. Geom. 9 (2006), 135-186, math.CA/0506328.

[29] Kitaev A.V., Non-Schlesinger deformations of ordinary differential equations with rational coefficients, J. Phys. A: Math. Gen. 34 (2001), 2259-2272, nlin.SI/0102019.

[30] Poberezhnyi V.A., General linear problem of the isomonodromic deformation of Fuchsian systems, Math. Notes 81 (2007), 529-542.

[31] Schäfke R., Confluence of several regular singular points into an irregular singular one, J. Dynam. Control Systems 4 (1998), 401-424.

[32] Sibuya Y., Simplification of a system of linear ordinary differential equations about a singular point, Funkcial. Ekvac. 4 (1962), 29-56.

[33] Sibuya Y., Perturbation of linear ordinary differential equations at irregular singular points, Funkcial. Ekvac. 11 (1968), 235-246.

[34] Yoshida M., Takano K., On a linear system of Pfaffian equations with regular singular points, Funkcial. Ekvac. 19 (1976), 175-189. 